ARTICLE

https://doi.org/10.1038/s41467-019-09735-4

\title{
Gut microbiome-derived phenyl sulfate contributes to albuminuria in diabetic kidney disease
}

Koichi Kikuchi et al."

Diabetic kidney disease is a major cause of renal failure that urgently necessitates a breakthrough in disease management. Here we show using untargeted metabolomics that levels of phenyl sulfate, a gut microbiota-derived metabolite, increase with the progression of diabetes in rats overexpressing human uremic toxin transporter SLCO4C1 in the kidney, and are decreased in rats with limited proteinuria. In experimental models of diabetes, phenyl sulfate administration induces albuminuria and podocyte damage. In a diabetic patient cohort, phenyl sulfate levels significantly correlate with basal and predicted 2-year progression of albuminuria in patients with microalbuminuria. Inhibition of tyrosine phenol-lyase, a bacterial enzyme responsible for the synthesis of phenol from dietary tyrosine before it is metabolized into phenyl sulfate in the liver, reduces albuminuria in diabetic mice. Together, our results suggest that phenyl sulfate contributes to albuminuria and could be used as a disease marker and future therapeutic target in diabetic kidney disease. 
$\mathrm{D}$ iabetic kidney disease (DKD) occurs in approximately $20-30 \%$ of all diabetic subjects and represents a major cause of end-stage renal disease (ESRD), cardiovascular events and death ${ }^{1,2}$. It is difficult to identify type 2 diabetes patients who are at risk of developing progressive DKD based only on measurements of glomerular filtration rate (GFR) and albuminuria $^{3}$. Specific biomarkers are needed for identifying individuals during the early stages of diabetes who are at high risk of complications ${ }^{2}$.

The major function of the kidney is the filtration or transport of various circulating endogenous and exogenous compounds into the urine ${ }^{4}$. Type 2 diabetes causes significant changes in an array of plasma metabolites, even in the early stages 5 . In the kidney, the organic anion transporting polypeptide (OATP) transporter family contributes to the transport of various organic anions and cations into the urine. In rodent kidney, several oatp gene products are present at the proximal tubules, whereas SLCO4C1 is the only OATP in human kidney ${ }^{4}$. The diversity among the oatp family members makes it difficult to extrapolate from experimental studies on rodents to humans. To overcome this issue, we generated transgenic rats overexpressing human SLCO4C1 in the proximal tubule ${ }^{6}$. These rats are an excellent model for evaluating the human kidney-specific elimination for metabolites and uremic toxins. We previously clarified that the excretion of uremic toxins in the SLCO4C1 transgenic rat model reduced hypertension, cardiomegaly, and inflammation in the setting of renal failure ${ }^{6}$.

Here, using this model, we characterize metabolites that are increased in diabetic wild-type rats (WT-DM), but reduced in diabetic SLCO4C1 transgenic rats (Tg-DM). We find that levels of phenyl sulfate (PS), a gut microbiota-derived metabolite, significantly correlate with basal and predicted 2-year progression of albuminuria in patients with microalbuminuria. Inhibition of PS production reduces albuminuria in diabetic mice. Together, our results suggest that PS is not only an early diagnosis marker, but also a modifiable cause and therefore a target for the treatment of DKD.

\section{Results}

Reduction of PS reduced proteinuria in a rat diabetes model. To identify metabolites linked to diabetic conditions, streptozotocin (STZ)-induced diabetes was induced in SLCO4C1-Tg rats on day 0 . On day 7 , bloods were collected and rats with blood glucose levels greater than $300 \mathrm{mg} / \mathrm{dl}$ were selected for further analysis. Dietary intake and body weights were similar in WTDM and SLCO4C1-Tg DM rats (Fig. 1). Blood glucose levels increased following STZ $(\sim 500 \mathrm{mg} / \mathrm{dl})$ and, during the experimental period, blood glucose levels were similar in both groups, except on day 90. The WT-DM and Tg-DM groups showed no differences in renal function. However, proteinuria in the Tg-DM group was significantly lower than in the WT-DM group on days 7,30 , and 63 , but showed a non-significant trend on days 90 and 119 (Fig. 1a). Histological analysis showed that glomerulomegaly was present in the WT-DM group, but reduced in the Tg-DM group (Fig. 1b). No significant change was observed in the preserved tubular and fibrotic areas between WT-DM and Tg-DM rats (Supplementary Fig. 1). These data suggest that overexpression of SLCO4C1 in diabetic kidney decreases proteinuria without major histological changes, except in glomeruli at the light microscopic level.

Identification of PS as a pathogen-derived metabolite in DKD. Since SLCO4C1 is a transporter that eliminates metabolites into urine, we concluded that excretion of a SLCO4C1-specific substrate into urine caused a reduction in proteinuria. Initially, we performed untargeted metabolome profiling to screen for major molecular differences using ultra high-performance liquid chromatography (UHPLC) coupled to quadrupole time-of-flight mass spectrometry (UHPLC-QTOF/MS). Over 4173 features were detected from the 5 groups: WT day 7 (WT-d7), WT day 63 (WT-d63), WT day 119 (WT-d119), SLCO4C1-Tg day 63 (Tgd63), and SLCO4C1-Tg day 119 (Tg-d119). Totally, 17.6\% (or 827 ) of the features with a coefficient of variation (CV) $\geq 30 \%$ were excluded and the remaining 3886 features were selected (Supplementary Data 1). Principle component analysis (PCA) was conducted on all groups (Supplementary Fig. 2a), all WT groups (days 7, 63, and 119, Supplementary Fig. 2b) and all TG groups (days 63 and 119, Supplementary Fig. 2c). Although some broad-based overlapping clustering can be observed, these analyses showed no clear distinction among the groups. This suggested that the largest source of variation is within each group and not among the groups.

To maximize and analyze differences among the five groups, partial least square-discriminant analysis (PLS-DA) was also performed. Figure 1c shows separation of the five groups analyzed. To evaluate the effect of STZ and highlight the most robust markers of interest, we used orthogonal PLS-DA (OPLSDA). We compared the plasma metabolomes between WT-d7 and WT-d119 rats, a time interval during which diabetic changes progressed (Fig. 1d). The OPLS-DA scores plot of WT-d7 and WT-d119 mice (Supplementary Fig. 2d) showed good fit and high predictability of the model with values for $R^{2} Y$ (which assesses the extent to which the data fit the model) and the quality assessment metric $Q^{2}$ of 0.994 and 0.956 , respectively. From the S-plot of the OPLS-DA ${ }^{7}, 94$ features that were significantly higher in WT-d119 also had a correlation value ( $p($ corr) $[1])>0.85$ (up to 1.0 ). These features were further filtered down to components with a low $\%$ CV $(<15 \%)$ (Supplementary Table 1$)$.

We next looked for analytes with high variable importance in projection (VIP) scores, which assess the relative contribution to the OPLS-DA model. These analyses identified one metabolite $(\mathrm{m} / z$ 172.97, red circle and arrow) that showed a distinctly strong contribution (high $\mathrm{p}[1]$ ) relative to the other features, with a fold change of 1.9, a distinct VIP score of 5.9 relative to the other components of VIP score $<1.5$ (Fig. 1d, Supplementary Table 1). Further investigation revealed that it increased with diabetes progression. This increase was significantly reduced in SLCO4C1Tg rats when compared to WT rats on days 63 and 119. This metabolite also showed a distinctly strong contribution relative to the other components in the S-plot of WT-d63 vs. Tg-d63 (Fig. 1e, red arrow) and the S-plot of WT-d119 vs. Tg-d119 (Fig. 1f). In summary, this feature was the only one to fulfill the selection criteria of increasing with the progression of diabetes and being reduced in $\mathrm{Tg}$ rats, and led to its selection for further investigation. The change in intensity of $m / z 172.97$ is shown in Fig. 1g. The fold changes and associated $p$ values for WT-d63 vs. Tg-d63 and for WT-d119 vs. Tg-d119 are listed in Supplementary Table 1.

To identify the feature, the elemental composition was analyzed and predicted to be $\mathrm{C}_{6} \mathrm{H}_{5} \mathrm{O}_{4} \mathrm{~S}\left([\mathrm{M}-\mathrm{H}]^{-}\right)$, with a theoretical value of $\mathrm{m} / \mathrm{z}$ 172.97. A search of the Chemspider database using the predicted elemental composition $\mathrm{C}_{6} \mathrm{H}_{5} \mathrm{O}_{4} \mathrm{~S}$ $\left([\mathrm{M}-\mathrm{H}]^{-}\right)$revealed the following candidates: 2-hydroxybenzenesulfonate, 3-hydroxybenzenesulfonate, 4-hydroxybenzenesulfonate, and PS. We tried to identify these features by matching fragments of the MS/MS spectra obtained from rat plasma. The product ions found in the corresponding extracted $\mathrm{MS}^{\mathrm{E}}$ highenergy spectrum at $\mathrm{m} / z \mathrm{z} 79.9, \mathrm{~m} / \mathrm{z} 93.0, \mathrm{~m} / \mathrm{z} 109.0$, and $\mathrm{m} / \mathrm{z} 121.0$

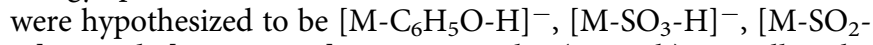
$\mathrm{H}]^{-}$, and $\left[\mathrm{M}-\mathrm{C}_{4} \mathrm{H}_{4}-\mathrm{H}\right]^{-}$, respectively (Fig. 1h). Finally, the identity was confirmed using a synthesized chemical standard of 
a
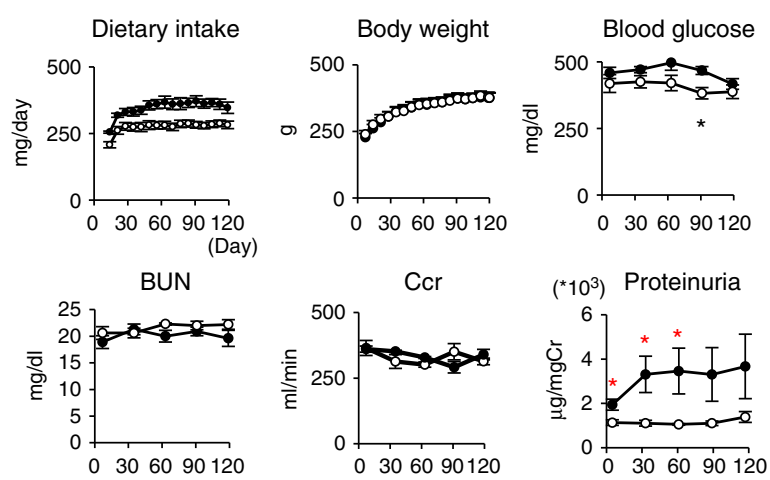

b
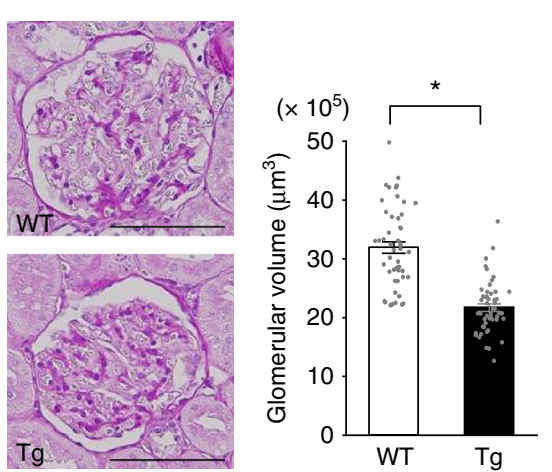

C

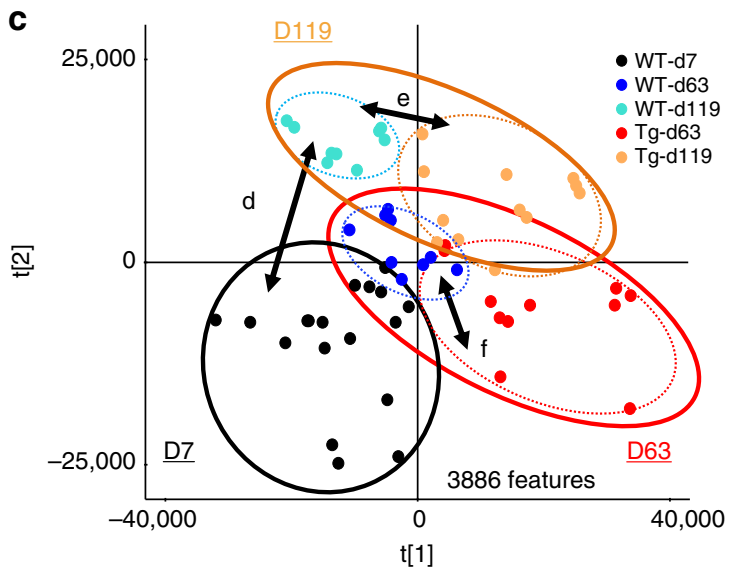

d

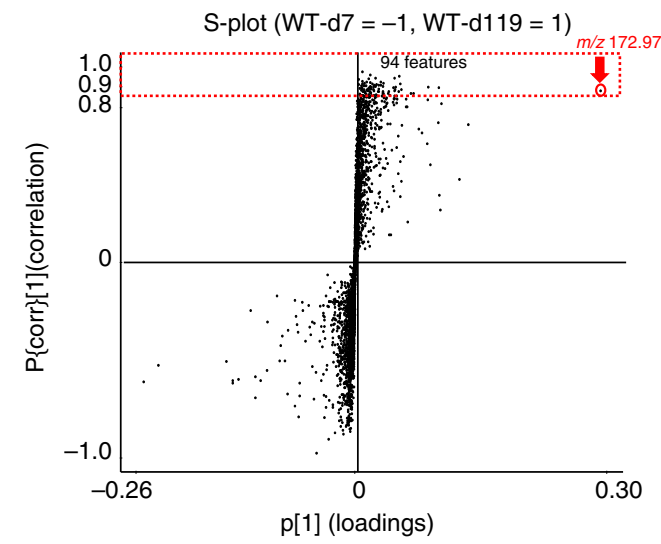

e
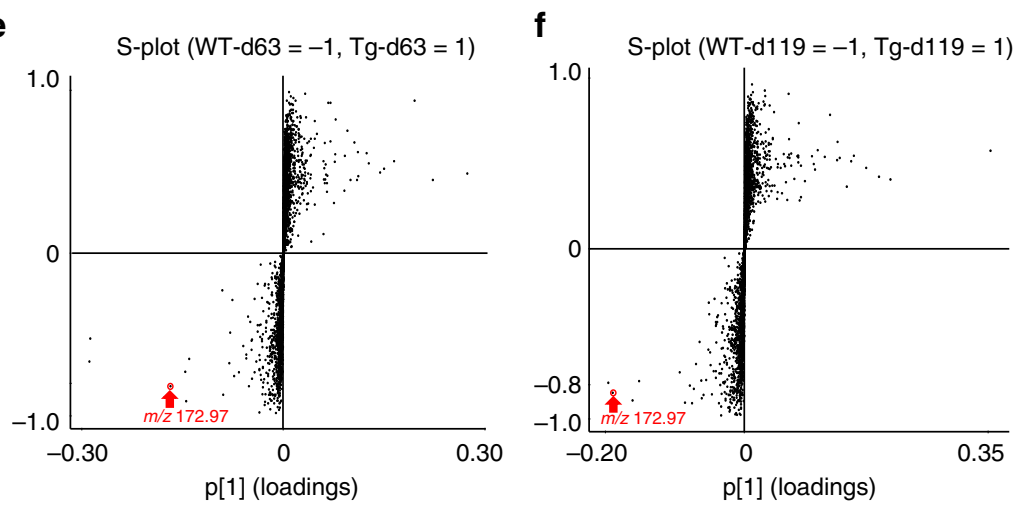

g

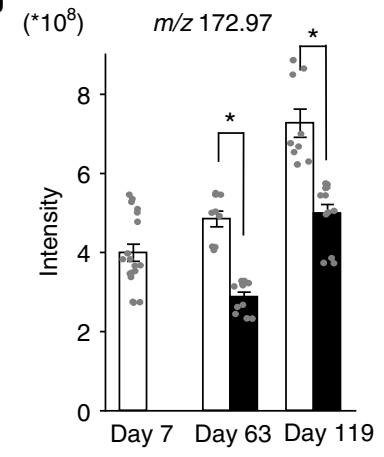

h

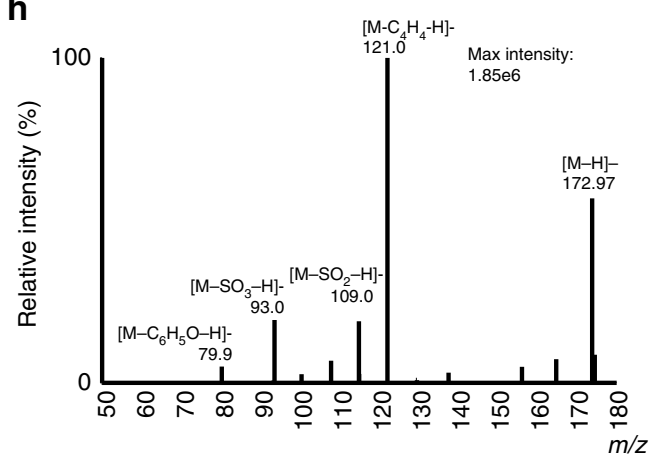

i<smiles>O=S(=O)(O)Oc1ccccc1</smiles>

j

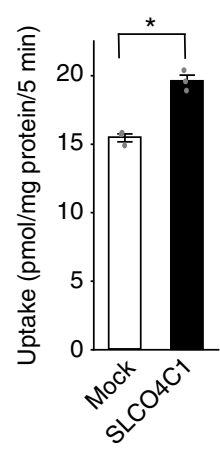

PS (Fig. 1i), which provided a similar fragmentation pattern and retention time. The plasma PS levels measured precisely by liquid chromatography (LC)/MS/MS ${ }^{8}$ in WT-DM rats on days 63 and 119 were $4.47 \pm 0.43$ and $6.48 \pm 0.71 \mu \mathrm{M}$, respectively, and these levels were decreased in Tg-DM rats (Supplementary Fig. 3). To confirm whether PS is a substrate of SLCO4C1, an uptake study was performed using SLCO4C1-expressing MDCK (SLCO4C1/ MDCKII) cells ${ }^{9}$. The uptake of PS into cells was increased in SLCO4C1/MDCKII cells, verifying PS as a substrate for the SLCO4C1 transporter (Fig. 1j). We also examined PS uptake using human proximal tubular HK-2 cells. The uptake of PS by HK-2 cells was inhibited by the SLCO4C1 inhibitor ritonavir 
Fig. 1 Diabetic SLCO4C1-Tg rats showed reduced proteinuria. a Body weight, blood glucose, blood urea nitrogen (BUN), and creatinine clearance (Ccr) in diabetic SLCO4C1-Tg rats (white circles, $n=5$ ) and WT rats (black circles, $n=6$ ). b Representative PAS (left) stained sections of WT and SLCO4C1-Tg rat glomeruli. Bars $=100 \mu \mathrm{m} . n=50$ in each group. Data are mean \pm SEM. ${ }^{*} p<0.05$ vs. WT. Student $t$ test. $\mathbf{c}$ Variation in the five groups in the PLS-DA scores plot using the chemical features detected in plasma. Sample conditions are represented by color coded circles: WT-d7 (black), WT-d63 (blue), WT-d119 (cyan), Tg-d63 (red), and Tg-d119 (orange). Groups D7, D63, and D119 are surrounded by black, red, and orange solid rings, respectively. Groups WT-d63, WT-d119, Tg-d63, and Tg-d119 are surrounded by blue, cyan, red, and orange dotted rings, respectively. $\mathbf{d}$ S-plot analyses of orthogonal partial least squarediscriminant analysis (OPLS-DA) of WT rats on days 7 and 119. The selected 94 features (surrounded by a dotted red rectangle) were significantly greater on day 119 than on day 7. e S-plot analyses of OPLS-DA of WT and Tg rats on day 63. $\mathbf{f}$ S-plot analyses of OPLS-DA of WT and Tg rats on day 119. $\mathbf{g}$ Changes in the concentration of the selected feature $\mathrm{m} / z 172.97$ with the progression of diabetes. Wild-type rats (white column, $n=6$ ); SLCO4C1-Tg rats (black column, $n=5$ ). $\mathbf{h}$ Fragment ion mass spectrum of $m / z$ 172.97. The main detected precursor and fragment ion was $m / z 172.97$. The product ions found in the corresponding extracted $\mathrm{MS}^{E}$ high-energy spectrum at $\mathrm{m} / \mathrm{z}$ 79.9, $\mathrm{m} / \mathrm{z}$ 93.0, $\mathrm{m} / \mathrm{z}$ 109.0, and $\mathrm{m} / \mathrm{z} 121.0$ were hypothesized to be $\left[\mathrm{M}-\mathrm{C}_{6} \mathrm{H}_{5} \mathrm{O}-\mathrm{H}\right]-$, $\left[\mathrm{M}-\mathrm{SO} \mathrm{O}_{3}-\mathrm{H}\right]-$ $\left[\mathrm{M}-\mathrm{SO}_{2}-\mathrm{H}\right]$-, and $\left[\mathrm{M}-\mathrm{C}_{4} \mathrm{H}_{4}-\mathrm{H}\right]$-, respectively. $\mathbf{i}$ Chemical structure of PS. $\mathbf{j}$ SLCO4C1-mediated PS uptake by SLCO4C1/MDCKII cells $(n=3)$. Data are mean \pm SEM. ${ }^{*} p<0.05$ vs. control according to Student $t$ test $(\mathbf{a}, \mathbf{b}, \mathbf{g}$, j). Source data are provided as a Source Data file and untargeted metabolome data are provided as a Supplementary Data 1

(Supplementary Fig. 4). These data demonstrated that PS increased with the progression of diabetes. Urinary protein and PS accumulated in diabetic rats were reduced by the overexpression of SLCO4C1 in the kidney.

PS-induced albuminuria by podocyte damage in diabetic models. We used several models to determine whether PS plays a pathogenic role in the progression of albuminuria and diabetic renal damage. The basal PS level in $\mathrm{db} / \mathrm{db}$ mice was $3.06 \pm$ $2.63 \mu \mathrm{M}$ (Fig. 2a, $n=5$ ). To accelerate phenotypic changes, we administered PS orally to two types of diabetes models. Oral administration of PS to $\mathrm{db} / \mathrm{db}$ mice $(50 \mathrm{mg} / \mathrm{kg})$ resulted in a rapid rise in plasma PS to $64.53 \pm 5.87 \mu \mathrm{M}$ in $1 \mathrm{~h}$, which then rapidly decreased ( $n=3$, Supplementary Fig. 5 ). After oral administration of PS to $\mathrm{db} / \mathrm{db}$ mice for 6 weeks, the plasma level was significantly increased by 5 -fold $(27.3 \pm 9.17 \mu \mathrm{M}$, Fig. $2 \mathrm{a})$. There was no change in the biological data between control $\mathrm{db} / \mathrm{db}$ mice and PS-treated $\mathrm{db} / \mathrm{db}$ mice (Supplementary Table 2).

Since PS is usually detected in renal failure and recognized as a gut-derived uremic toxin ${ }^{10}$, we measured the plasma level of other gut microbiota-derived uremic toxins ( $p$-cresyl sulfate (PCS), indoxyl sulfate (IS), and trimethylamine $N$-oxide (TMAO), Fig. 2a). We found no significant differences in PCS, IS, and TMAO concentrations following PS administration. Albuminuria was increased in $\mathrm{db} / \mathrm{db}$ mice supplemented with PS (Fig. 2b).

Because podocyte injury causes albuminuria ${ }^{11}$, we focused on the effect of PS on podocytes. Histological examination by light microscopy revealed mesangial expansion, but not significant (Fig. 2c). However, electron microscopy revealed increased the foot processes effacement (Fig. 2c, white arrows) and the glomerular basement membrane thickening (GBM) (Fig. 2c, yellow arrow heads) in PS-treated $\mathrm{db} / \mathrm{db}$ mice. These data suggest that PS elicits podocyte damage in diabetic mice. In addition, although there was no difference between in the preserved tubular area and fibrotic area at the light microscopic level (Supplementary Fig. 6a), the expression levels of representative inflammatory genes, TNF- $\alpha(\operatorname{Tnf} a)$ and MCP-1 (Ccl2), the fibrotic gene TGF- $\alpha_{1}$ $(T g f b 1)$, fibronectin $(F n 1)$, and collagen I (Colla1) were increased in PS-treated $\mathrm{db} / \mathrm{db}$ kidney (Supplementary Fig. 6b). It was reported that $\mathrm{db} / \mathrm{db}$ mice develop renal fibrosis ${ }^{12}$. Our data further suggest pro-fibrotic and pro-inflammatory effects of PS in the DKD model.

To confirm the toxic effect of PS on podocytes, we employed cell survival analysis using differentiated human urinary podocyte-like epithelial cells (HUPECs) ${ }^{13}$. As shown in Fig. 2d, PS was toxic to differentiated podocytes from $30 \mu \mathrm{M}$ and significant cell toxicity was seen at concentrations from $100 \mu \mathrm{M}$. We also measured the effect of PS on glutathione levels, as it was reported PS decreases glutathione levels, rendering the cells vulnerable to oxidative stress ${ }^{14}$. Glutathione levels in differentiated podocytes exposed to PS $(30 \mu \mathrm{M})$ decreased and significant at $100 \mu \mathrm{M}$ (Fig. 2e). This was comparable to reports in CKD $(\sim 62 \mu \mathrm{M})^{15}$ and in our human DKD study $(0-68.1 \mu \mathrm{M}$, Table 1$)$.

Since podocyte mitochondrial dysfunction was also reported in the pathogenesis of $\mathrm{DKD}^{16}$, we examined the bioenergetic effect of PS on cultured podocytes ${ }^{17}$. By measuring the oxygen consumption rate (OCR), PS $(100 \mu \mathrm{M}$ to $1 \mathrm{mM})$ exposure to differentiated podocytes significantly decreased mitochondrial basal respiration, ATP production, $\mathrm{H}^{+}$leaking and maximum respiration capacity, suggesting that PS is toxic to mitochondria (Fig. 2f and Supplementary Fig. 7). PS also increased nonmitochondrial respiration, suggesting respiration compensation. Extracellular acidification rate (ECAR) measuring glycolysis did not differ between PS-treated and control differentiated podocytes, further suggesting that glycolysis was not compensated enough.

To confirm the effect of PS in diabetic kidney, we also administered PS $(50 \mathrm{mg} / \mathrm{kg})$ to KKAy mice fed on a high-fat diet (HFD), another model of $\mathrm{DKD}^{18}$. The plasma PS level was significantly increased to $6.09 \pm 3.18 \mu \mathrm{M}(n=4)$ in PS-treated HFD-KKAy mice compared to non-PS-treated HFD-KKAy mice $(1.48 \pm 0.85 \mu \mathrm{M}$; Fig. $2 \mathrm{~g}, n=3))$. The clinical data had not changed (Supplementary Table 3). Oral administration of PS to HFD-KKAy mice for 6 weeks increased albuminuria (Fig. $2 \mathrm{~g}$ ). Electron micrographs revealed podocyte effacement (Fig. $2 \mathrm{~h}$, white arrows) and GBM thickening (Fig. 2h, yellow arrow heads) and damage in the PS-treated HFD-KKAy kidney. Elastica Masson and F4/80 staining showed some perivascular fibrotic area in the PS-treated group (Fig. $2 \mathrm{~h}$, black arrow heads). These results further suggest that the manifested podocyte damage and perivascular inflammation were elicited by PS, which contributed to albuminuria in HFD-KKAy mice.

We further examined diabetic mice lacking endothelial nitric oxide synthase (eNOS) by introducing the Akita mutation in the insulin2 (Ins2) gene ${ }^{19}$. This is a model of severe diabetes that recapitulates human DKD with mesangial expansion, mesangiolysis, glomerulosclerosis, and GBM thickening ${ }^{19}$. In this model, blood glucose level of eNOS-KO mice was $150 \pm 15 \mathrm{mg} / \mathrm{dl}$ and this level was significantly increased at $460 \pm 45 \mathrm{mg} / \mathrm{dl}$ at 6 months $(n>5)$. The urinary albumin level was also increased in eNOSKO-DM mice $(413 \pm 107 \mu \mathrm{g} / \mathrm{mgCr})$ compared to that in eNOS$\mathrm{KO}$ mice $(36 \pm 4 \mu \mathrm{g} / \mathrm{mgCr})^{19}$. The plasma PS level in eNOS-KODM mice was significantly increased $(15.4 \pm 2.73 \mu \mathrm{M}, n=5)$ compared with control eNOS-KO mice (5.97 $\pm 0.47 \mu \mathrm{M}$, Fig. $2 \mathrm{i})$, although the renal function in both DM and non-DM mice was normal. Histological examination revealed that NOS-KO-DM diabetic mice showed significant changes in glomerulosclerosis 
a
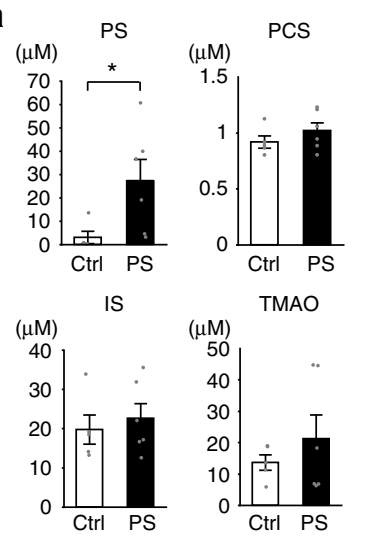

d

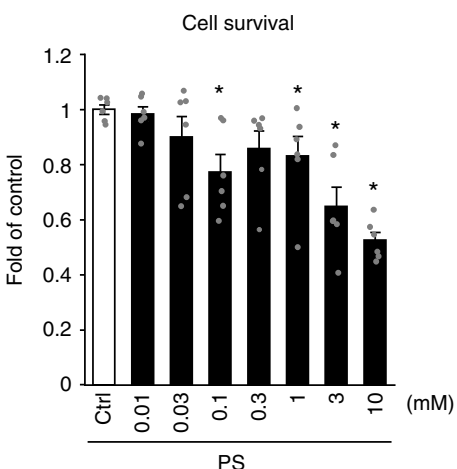

e

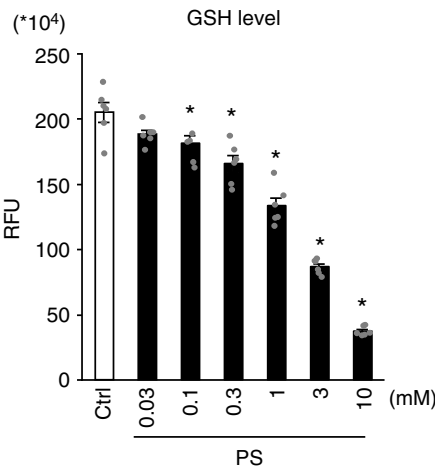

C

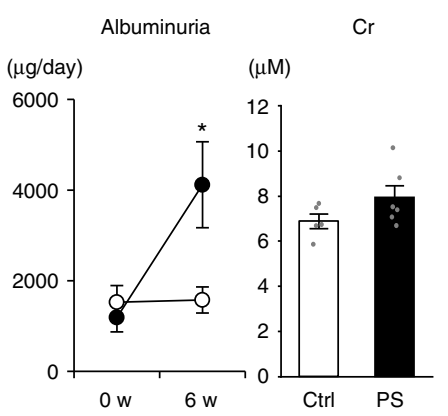

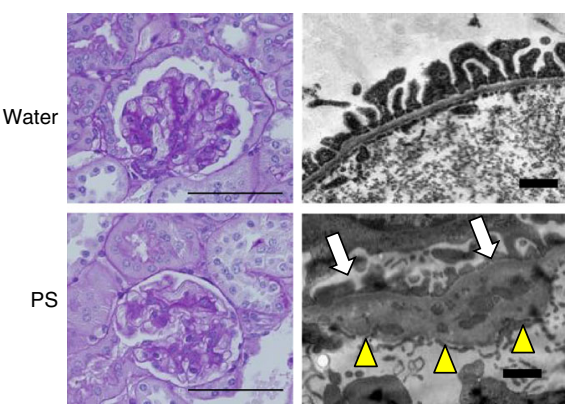

f ATP production Proton leak Max respiratio

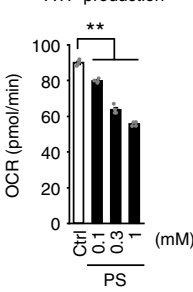

Basal respiration

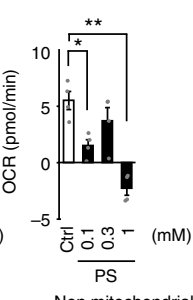

120 **
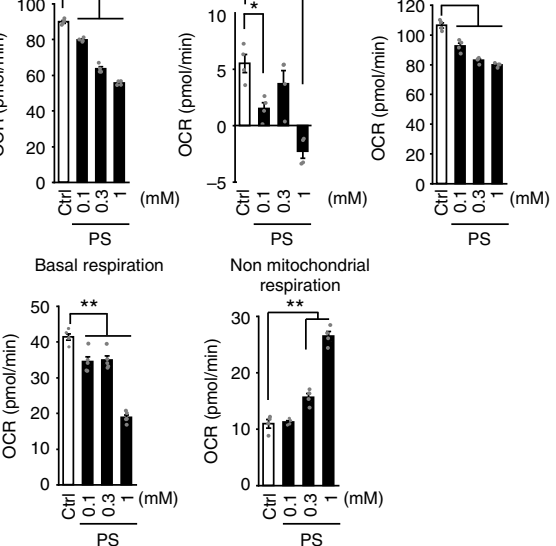

Non mitochondria

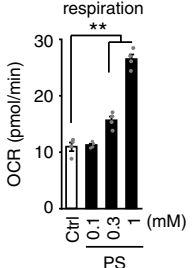

g

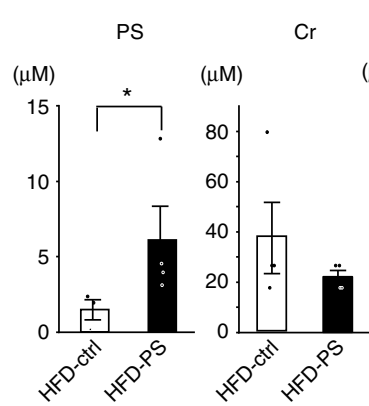

i

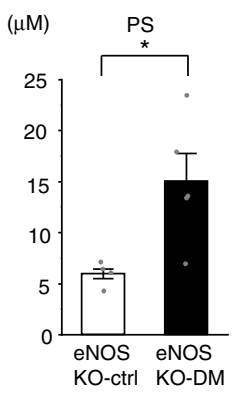

Albuminuria

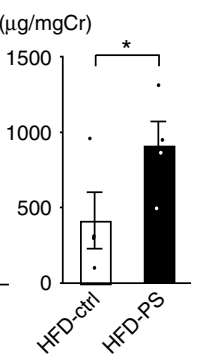

h

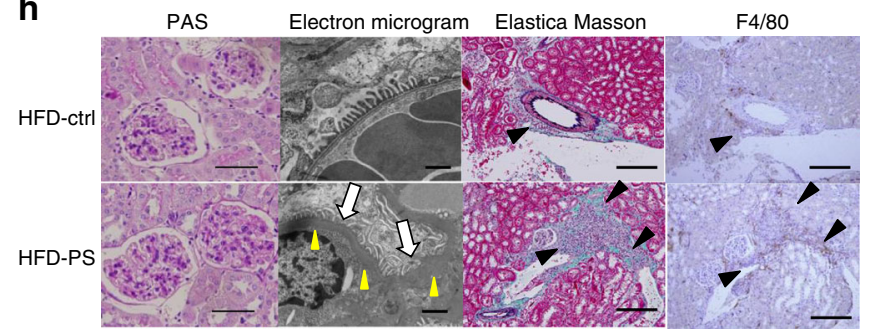

j
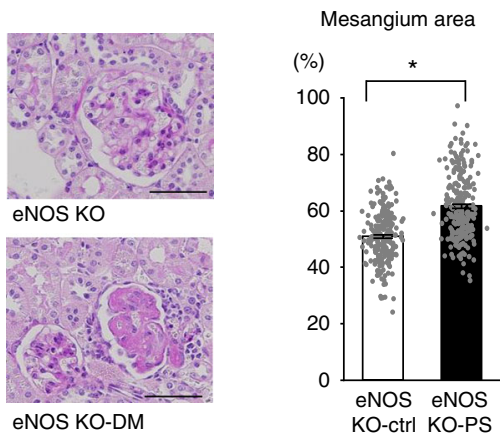

compared with NOS-KO-control mice (Fig. 2j). These data further suggested that a higher PS level shows much severer glomerular lesion.

PS is a predictor for incipient albuminuria in DKD patients. To confirm the relationship between PS and albuminuria observed in animal experiments, we recruited 362 diabetes patients from the U-CARE (urinary biomarker for continuous and rapid progression of diabetic nephropathy) study ${ }^{20}$. We analyzed two parameters: (i) the relationship between the baseline PS and albuminuria and (ii) the relationship between the baseline PS concentration and the progression of albuminuria up until 2 years later. We also measured soluble urokinase-type plasminogen activator receptor (suPAR), which associated with the 
Fig. 2 Pathophysiological features of PS in vitro and in vivo. a Plasma levels of PS, PCS; IS, and TMAO in 11-week-old db/db mice in control (Ctrl; $n=5)$ or PS ( $50 \mathrm{mg} / \mathrm{kg} /$ day for 6 weeks, $n=6$ ). b Plasma albumin and Cr levels before and after administering PS for 6 weeks to db/db mice. Control (white circles, $n=5$ ) and PS-treated (black circles, $n=6$ ) groups. c Glomeruli in $\mathrm{db} / \mathrm{db}$ mouse with or without PS for 6 weeks. Bar, $80 \mu \mathrm{m}$ (PAS) and $1 \mu \mathrm{m}$ (EM). d Cell toxicity analysis $(n=6)$. e Cellular GSH level $(n=6)$. $\mathbf{f}$ Bioenergetic characterization of cultured human podocytes in terms of oxygen consumption rate (OCR). $n=4$. g PS, albuminuria and Cr levels before and after administering PS for 6 weeks in HFD-KKAy mouse ( 6 weeks old). For PS, $n=3$ (HFDcontrol) and $n=4$ ((HFD-PS). For $\mathrm{Cr}$ and albuminuria, $n=4$. Wilcoxon (PS) and Student's $t$ test ( $\mathrm{Cr}$ and albuminuria). $\mathbf{h}$ Histological images of PAS and electron microscopic analysis of podocytes from control (Ctrl; top row) and PS-treated (PS; bottom row) groups of HFD-KKAy mice. Scale bar $=200 \mu \mathrm{m}$ for PAS and $1 \mu \mathrm{m}$ for electron microscopy. The effacement of podocytes (white arrows) and GBM thickness (yellow arrow heads) are shown. The inflammatory area (Elastica Masson) and macrophage infiltration (F4/80 immunostaining) around the vascular area are indicated (black arrow heads). Scale bar, $80 \mu \mathrm{m}$. i PS levels in eNOS knockout mice with or without diabetes $(n=5)$. j Histological examination stained with PAS of eNOS knockout mice with or without diabetes. Scale bar, $50 \mu \mathrm{m}$. ${ }^{\star} p<0.05$ vs. control according to Student $t(\mathbf{a}, \mathbf{b}, \mathbf{d}, \mathbf{e}, \mathbf{I}, \mathbf{j})$ or Tukey's test (f). Source data are provided as a Source Data file

\begin{tabular}{|c|c|c|c|}
\hline & & Baseline & After 2 years \\
\hline $\begin{array}{l}\text { PS } \\
\text { SUPAR }\end{array}$ & $\begin{array}{l}(\mu \mathrm{M}) \\
(\mathrm{pg} / \mathrm{mL})\end{array}$ & $\begin{array}{l}3.3(0-68.1) \\
460.8 \\
(142.0-2740.2)\end{array}$ & \\
\hline Age & (years) & $63.3 \pm 12.4$ & $65.3 \pm 12.4$ \\
\hline Gender & $(\mathrm{M} / \mathrm{F})$ & $206 / 156$ & $206 / 156$ \\
\hline DM type & (1/2/others) & $40 / 318 / 4$ & \\
\hline Duration & (years) & $14.6 \pm 8.8$ & $16.6 \pm 8.8$ \\
\hline $\begin{array}{l}\text { Time to } \\
\text { measure }\end{array}$ & (years) & & $2.08 \pm 0.18$ \\
\hline BMI & $\left(\mathrm{kg} / \mathrm{m}^{2}\right)$ & $25.0 \pm 4.4$ & \\
\hline SBP & $(\mathrm{mmHg})$ & $127.8 \pm 15.2$ & $128.6 \pm 15.8$ \\
\hline DBP & $(\mathrm{mmHg})$ & $72.4 \pm 10.2$ & $72.1 \pm 10.6$ \\
\hline BS & $(\mathrm{mg} / \mathrm{dL})$ & $154.2 \pm 56.4$ & $159.5 \pm 60.6$ \\
\hline HbA1c & (\%) & $7.2 \pm 1.1$ & $7.3 \pm 1.1$ \\
\hline eGFR & $\begin{array}{l}(\mathrm{mL} / \mathrm{min} / \\
\left.1.73 \mathrm{~m}^{2}\right)\end{array}$ & $73.8(17.1-115.4)$ & $\begin{array}{l}72.6 \\
(13.8-112.4)\end{array}$ \\
\hline$A C R$ & $(\mathrm{mg} / \mathrm{gCr})$ & $11.0(1.0-6407.4)$ & $13.1(1.1-3410.8)$ \\
\hline ALT & $(I U / L)$ & $22.5 \pm 12.5$ & $23.4 \pm 14.9$ \\
\hline TC & $(\mathrm{mg} / \mathrm{dL})$ & $175.4 \pm 31.6$ & $179.9 \pm 32.1$ \\
\hline TG & $(\mathrm{mg} / \mathrm{dL})$ & $132.2 \pm 82.6$ & $133.1 \pm 79.4$ \\
\hline $\mathrm{HDL}$ & $(\mathrm{mg} / \mathrm{dL})$ & $55.3 \pm 16.6$ & $57.1 \pm 16.7$ \\
\hline UA & $(\mathrm{mg} / \mathrm{dL})$ & $5.3 \pm 1.7$ & $5.1 \pm 1.4$ \\
\hline
\end{tabular}

Baseline and 2-year after characteristics of the U-CARE study ( $n=362$ DKD patients). Mean \pm SD was for parametric factors and the median range was for nonparametric factors (eGFR, ACR, PS, and suPAR). In the U-CARE study, eGFR (CKD-EPI) is the estimated eGFR based on the equation of Chronic Kidney Disease Epidemiology Collaboration (CKD-EPI). PS phenyl sulfate concentration, sUPAR soluble urokinase-type plasminogen activator receptor, duration duration of diabetic mellitus, $B M I$ body mass index, SBP systolic blood pressure, $D B P$ diastolic blood pressure, BS blood glucose level, HbA1c NGSP value, eGFR estimated glomerular filtration rate (CKD-EPI), ACR urinary albumin-to-creatinine ratio, ALT alanine aminotransferase, TC total (CKD-EPI), ACR urinary albumin-to-creatinine ratio, ALT alanine aminotransferase, TC to
cholesterol, TG triglyceride, $H D L$ high-density lipoprotein cholesterol, UA uremic acid.

incidence and progression of DKD by modification of podocyte barrier function ${ }^{21,22}$.

Participants in the U-CARE study $(n=362)$ had a mean age of 63.3 years, and $56.9 \%$ were male. The mean blood glucose concentration (BS) was $154.2 \pm 56.4 \mathrm{mg} / \mathrm{dl}$ and the $\mathrm{HbAlc}$ was $7.2 \pm 1.1 \%$. The eGFR was $73.8(17.1-115.4) \mathrm{ml} / \mathrm{min} / 1.73 \mathrm{~m}^{2}$ and the albumin-to-creatinine ratio (ACR) was $11.0(1.0-6407.4) \mathrm{mg} /$ gCr. The serum PS level was $3.3 \mu \mathrm{M}(0-68.1 \mu \mathrm{M})$ and suPAR was 460.8 (142-2740.2) $\mathrm{pg} / \mathrm{ml}$ (Table 1). Supplementary Table 4 shows clinical data for patients with DKD based on the albuminuria and Fig. 3a shows the PS level.

In the Spearman rank-order test (Supplementary Table 5), the plasma PS level significantly correlated with ACR (Fig. 3b), eGFR (Fig. 3c), age, duration, HbAlc, and uric acid (UA), but not with suPAR (Fig. 3d). We next performed multiple regression analysis. To evaluate, we built three models: model 1 (crude model); model 2 (adjusted by known factors: age, gender, BMI,
SBP, HbAlc, log (eGFR) ${ }^{23}$; and model 3 (full model; adjusted by model 2 plus other clinical factors in Supplementary Table 4, i.e., DBP, ALT, total cholesterol (TC), triglyceride (TG), high-density lipoprotein (HDL) and UA). Multiple regression analysis revealed that ACR was the only factor that significantly correlated with PS among all models (Table 2). Stepwise analysis for progression using known factors indicated the significance of the same factors. In addition, vice versa, analysis performed with ACR as an objective variable revealed that PS significantly correlated with the ACR among all models as well as stepwise analysis (Table 3). In this analysis, eGFR, gender, and blood pressure were also related to the ACR, as reported ${ }^{24}$, suggesting the validity of our cohort.

Given the significant relationship between baseline PS and albuminuria, we examined logistic regression models to identify factors independently associated with the development of the 2-year ACR (Table 4). The mean follow-up duration was $2.1 \pm 0.2$ years (median $=2.1$ years, interquartile range: $2.0-2.2$ years). Based on model 1, PS and suPAR were the only factors related to the amount of change in the ACR during the 2 years, suggesting that PS and suPAR are independent risk factors for the ACR. In models 2 and 3, PS and suPAR were not significant. However, receiver operating characteristic (ROC) curve analysis revealed that the $c$-statistics value-the areas under the curve ROC (AUCROC) using known factors ${ }^{23}$ was 0.674 and the combination of PS increased the c-statistics value to 0.708 (Supplementary Fig. 8a). A combination of suPAR with known factors did not increase the $c$-statistics value from 0.674 to 0.664 (Supplementary Fig. 8b). However, the combination of PS with known factors, as well as suPAR, further increased the $c$-statistics value from 0.664 to 0.702 (Supplementary Fig. 8c).

Based on this, we performed stratified logistic analysis based on the disease stage. Owing to the insufficient number of macroalbuminuria patients for analysis, we examined the normoalbuminuria and microalbuminuria groups (Supplementary Table 6). In the normoalbuminuria group, PS was not related to the amount of change in the ACR during the 2 years in all models (Supplementary Table 6). On the other hand, in the microalbuminuria group, PS was the only factor related to the amount of change in the 2-year ACR in all three models, with an odds ratio of 2.02 (CI: 1.04-3.92) in model 2 (Fig. $3 \mathrm{e}$ and Supplementary Table 6). Stepwise model also indicated the significance of PS. However, suPAR was not related in either the normoalbuminuria or macroalbuminuria groups. ROC curve analysis also showed that the $c$-statistics value using known factors was 0.713 and the combination of PS increased this to 0.751 (Fig. 3f). A combination of suPAR with known factors also increased the $c$-statistics value from 0.713 to 0.725 (Fig. $3 g$ ). Further combination of PS with known factors and suPAR, still increased the c-statistics value from 0.725 to 0.752 (Fig. $3 \mathrm{~h}$ ). These data suggested that PS was related to ACR and that the PS 

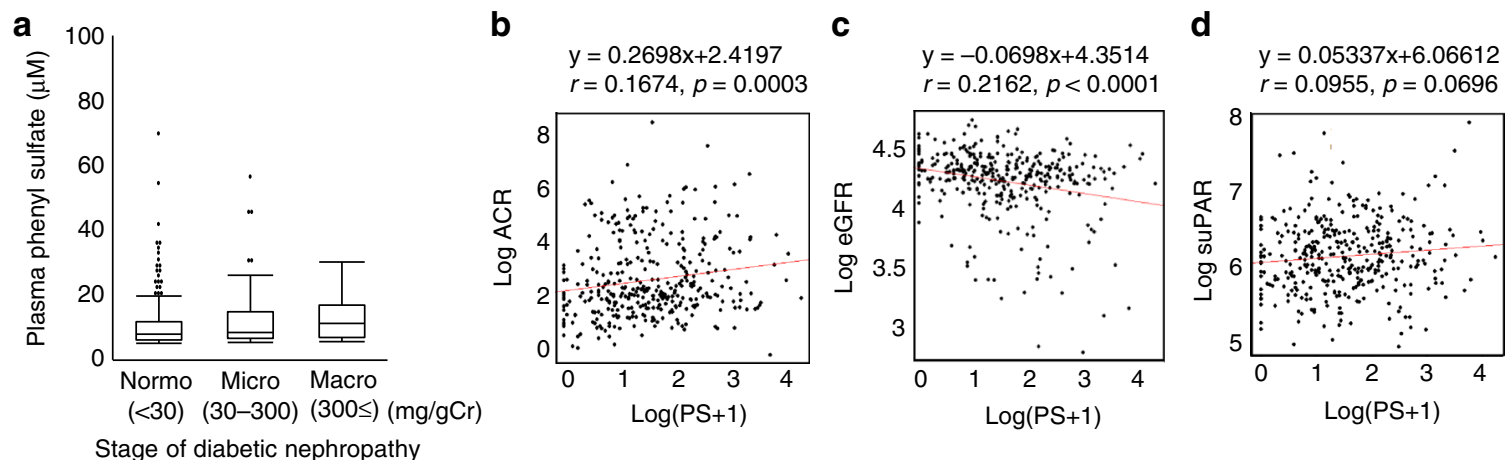

e

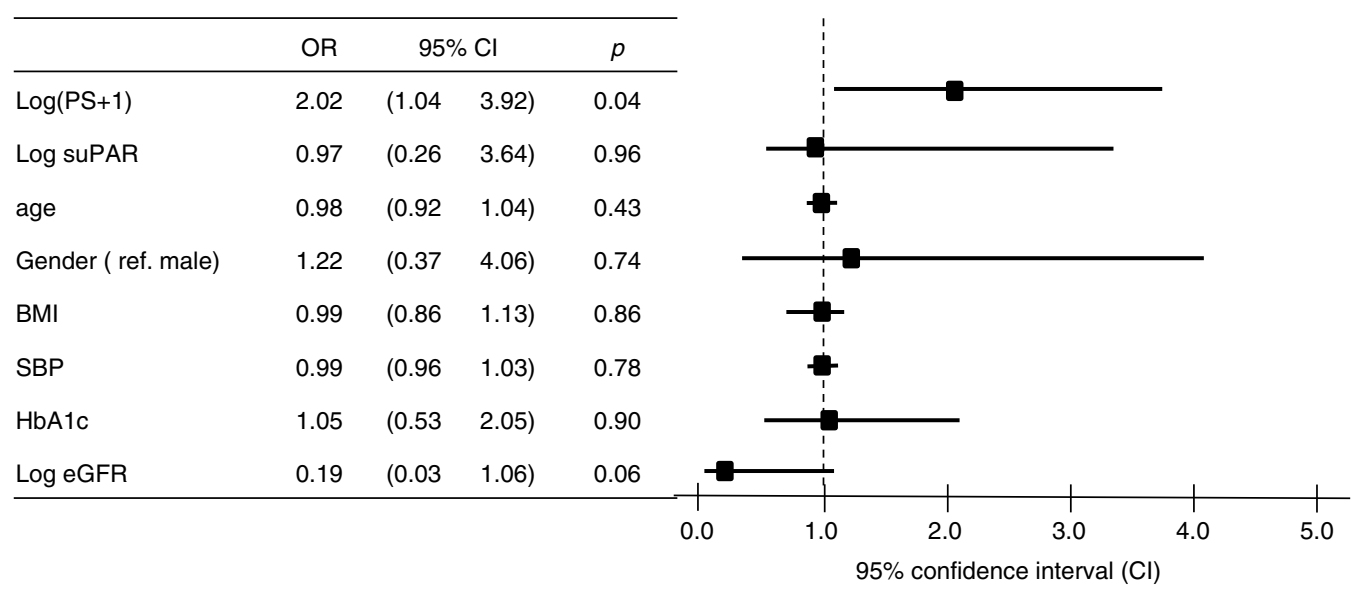

f

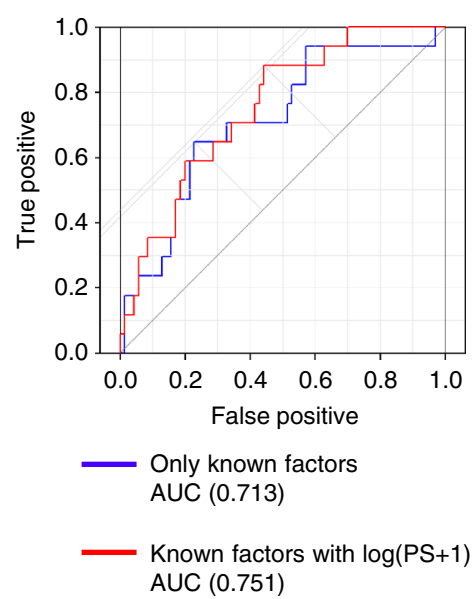

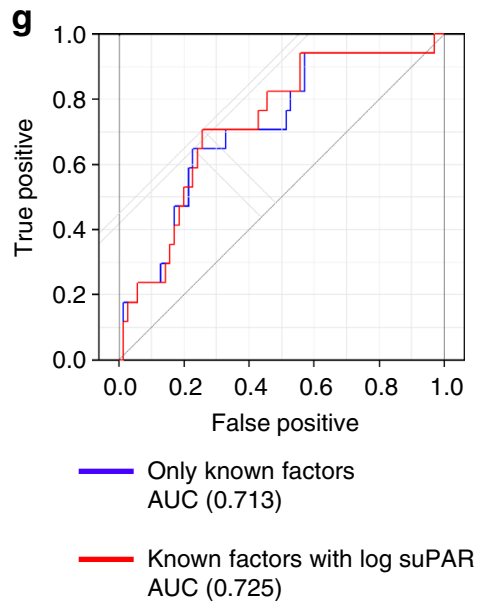

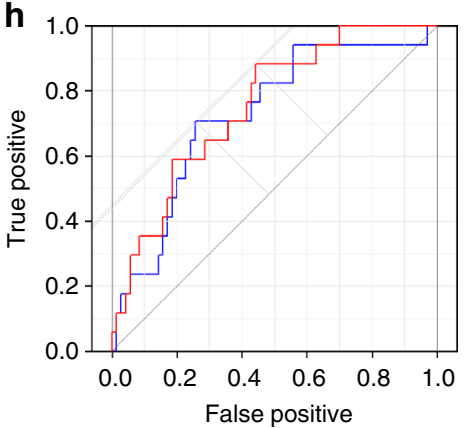

Known factors with log suPAR AUC (0.725)

Known factors with log suPAR with $\log (P S+1) A \cup C$ (0.752)

Fig. 3 The clinical significance of PS in DKD patients. a The relationship between the plasma PS concentration $(n=362)$. $\mathbf{b}$ The relationship between the plasma PS concentration and the urinary albumin level $(n=362)$. c The relationship between the plasma PS concentration and the eGFR $(n=362)$. $\mathbf{d}$ The relationship between the plasma PS concentration and soluble urokinase-type plasminogen activator receptor (suPAR) ( $n=362)$. e PS is a predictor of 2 year albuminuria. Adjusted odds ratio (95\% confidence interval). Note that, among the known factors ${ }^{23}$, PS was the only factor that served as a predictor of the progression of 2-year ACR in patients with microalbuminuria $(n=87)$. Receiver operating characteristic (ROC) curve analysis in the U-CARE Study, showing comparison of known factors ${ }^{23}$. $\mathbf{f}$ AUC-ROC using known factors (0.713) and adjusted with PS (0.751) ( $\left.n=87\right)$. $g$ AUC-ROC using known factors (0.713) and adjusted with suPAR (0.725). $(n=87)$. h AUC-ROC using known factors with suPAR $(0.725)$ and adjusted with PS (0.752) ( $n=87)$

concentration could predict the 2-year ACR deterioration in DKD patients, especially with microalbuminuria.

Microbial enzymatic reduction of PS showed reno-protection. Nonlethal inhibition of microbial-specific enzymes has a therapeutic advantage, with lower selective pressure for the development of drug resistance ${ }^{25}$. Phenol is synthesized from dietary L-tyrosine by gut bacterial-specific tyrosine phenol-lyase
(TPL, EC 4.1.99.2) ${ }^{26}$ and absorbed phenol is metabolized in to PS in the liver (Fig. 4a). TPL was reported to be expressed only in a minor population of Enterobacteriaceae (e.g., Citrobacter, Proteus, and Erwinia) ${ }^{26}$ and is not present in eukaryotes. Therefore, the idea arose that TPL inhibition might decrease the plasma PS level without any host toxicity. We synthesized the TPL inhibitors 2aza-tyrosine $^{27}$ and L-meta-tyrosine ${ }^{28}$ was purchased. We then examined their effects on the plasma PS level, albuminuria, and renal function in a diabetic model. Oral administration of 
Table 2 Multiple regression analysis (objective value: $(P S+1))$

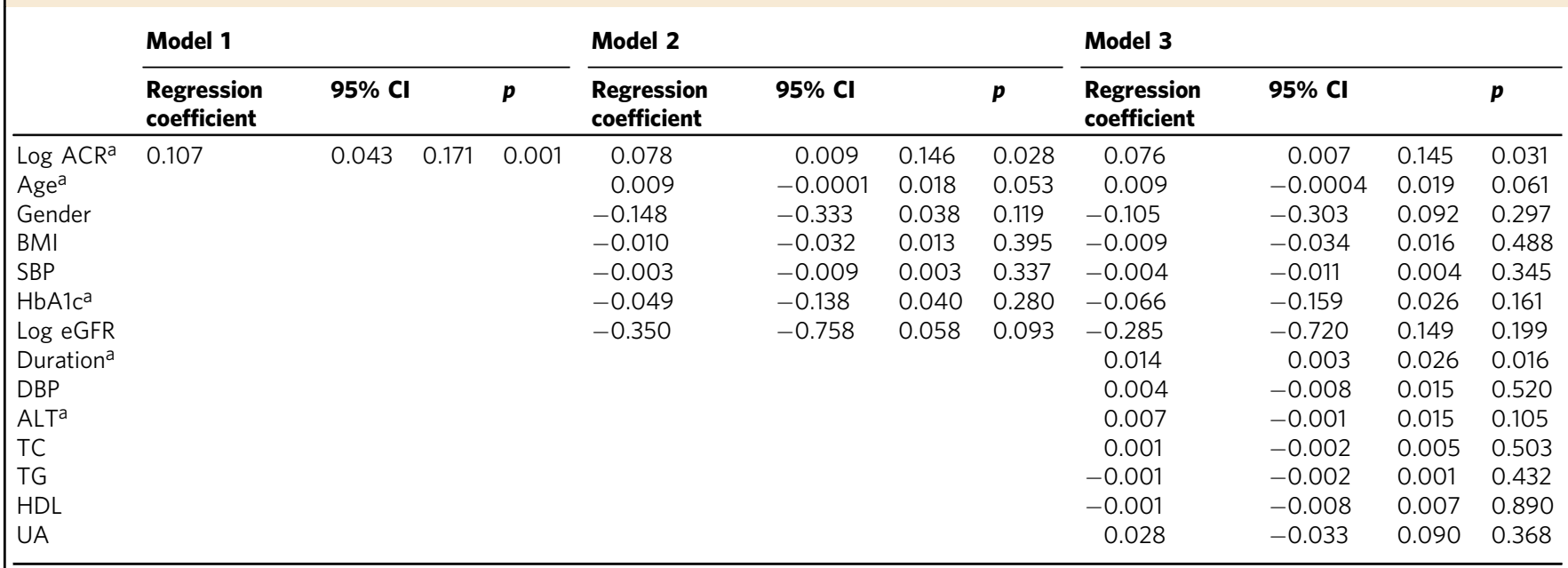

Multiple regression analysis based on clinical factors as an independent examined by variance inflation factor (VIF) <10. PS was used as an independent variable factor. Model 1 (crude model), model 2 (adjusted by known factors: age, gender, BMI, SBP, HbA1c, log (eGFR)) 23, and model 3 (full model, adjusted by model 2 plus fundamental clinical data in Supplementary Table 4 (DBP, ALT, TC, TG, HDL, and UA) were used.

${ }^{\star} p<0.05$ was regarded as statistically significant. Remaining variables after the stepwise method based on Akaike's information criterion (AIC) in model 3 are depicted as ${ }^{\star}$

Table 3 Multiple regression analysis (objective value: Log ACR)

\begin{tabular}{|c|c|c|c|c|c|c|c|c|c|c|c|c|}
\hline & \multicolumn{4}{|l|}{ Model 1} & \multicolumn{4}{|l|}{ Model 2} & \multicolumn{4}{|l|}{ Model 3} \\
\hline Age & & & & & -0.003 & -0.017 & 0.011 & 0.652 & -0.0004 & -0.015 & 0.014 & 0.961 \\
\hline Gender $^{\star}$ & & & & & 0.333 & 0.056 & 0.611 & 0.019 & 0.396 & 0.097 & 0.695 & 0.010 \\
\hline BMI & & & & & 0.020 & -0.013 & 0.054 & 0.233 & 0.017 & -0.022 & 0.055 & 0.397 \\
\hline Log eGFR ${ }^{\star}$ & & & & & -1.919 & -2.502 & -1.335 & $<0.001$ & -1.883 & -2.516 & -1.250 & $<0.001$ \\
\hline Duration & & & & & & & & & -0.003 & -0.021 & 0.015 & 0.716 \\
\hline $\mathrm{DBP}^{\star}$ & & & & & & & & & 0.010 & -0.007 & 0.027 & 0.243 \\
\hline ALT & & & & & & & & & -0.004 & -0.016 & 0.009 & 0.579 \\
\hline TC & & & & & & & & & -0.001 & -0.006 & 0.005 & 0.761 \\
\hline TG & & & & & & & & & 0.0004 & -0.002 & 0.002 & 0.692 \\
\hline $\mathrm{HDL}$ & & & & & & & & & 0.002 & -0.010 & 0.013 & 0.795 \\
\hline
\end{tabular}

2-aza-tyrosine $(10 \mathrm{mg} / \mathrm{kg} /$ day $)$ for 1 week significantly decreased plasma PS, IS, and PCS in db/db mice (Fig. 4b). The increased albuminuria observed during the progression of diabetes was reduced by 2 -aza-tyrosine (Fig. 4c, $10 \mathrm{mg} / \mathrm{kg} /$ day for 2 weeks). Furthermore, it was clearly demonstrated that the plasma creatinine (Cr) level was reduced by 2 -aza-tyrosine (Fig. $4 \mathrm{~b}$ ), suggesting a renoprotective effect. Similarly, another TPL inhibitor, L-meta-tyrosine, also reduced the plasma PS and IS levels, although the Cr level was not significantly changed in $\mathrm{db} / \mathrm{db}$ mice (Supplementary Fig. 9). These data suggested that 2-aza-tyrosine reduced not only albuminuria, but also the PS and IS levels, with a renoprotective effect in diabetic mice.

To confirm this renoprotective potency, we administered 2aza-tyrosine to a mouse model of adenine-induced renal failure ${ }^{29}$. In this model, the plasma PS, IS, PCS, and TMAO levels were significantly increased (Fig. 4d). By administering 2-aza-tyrosine
( $5 \mathrm{mg} / \mathrm{kg} /$ day), the plasma Cr level was significantly decreased, with plasma PS and IS levels showing a strong tendency to decrease, but not statistically significant. Tissue PS and IS contents are significantly increased in the renal cortex of kidney failure mice shown by matrix-assisted laser desorption/ionization (MALDI) mass spectrometry imaging (MSI) ${ }^{30}$. Imaging also revealed that the tissue PS and IS levels were decreased by 2-azatyrosine treatment (Fig. 4e). The quantitative tissue contents analysis by LC/MS/MS clearly revealed that the PS, IS, PCS, and TMAO levels were increased by renal failure and that the accumulation of PS, IS, and TMAO were significantly reduced by 2-aza-tyrosine (Fig. 4f). Furthermore, the tubular area damaged by renal failure was improved by 2 -aza-tyrosine (Fig. $4 \mathrm{~g}$ ). It is unclear how 2-aza-tyrosine reduced TMAO in renal failure tissue, but the amelioration of renal failure by 2 -aza-tyrosine may have, in part, contributed to these beneficial phenomena. 
Table 4 Logistic regression analysis

\begin{tabular}{|c|c|c|c|c|c|c|c|c|c|c|c|c|}
\hline \multirow[b]{3}{*}{$\log (P S+1)$} & \multicolumn{4}{|c|}{ Model 1} & \multicolumn{4}{|c|}{ Model 2} & \multicolumn{4}{|c|}{ Model 3} \\
\hline & \multirow{2}{*}{$\begin{array}{l}\text { Odds } \\
1.42\end{array}$} & \multicolumn{2}{|c|}{$95 \% \mathrm{Cl}$} & \multirow{2}{*}{$\begin{array}{l}\boldsymbol{p} \\
0.040\end{array}$} & \multirow{2}{*}{$\begin{array}{c}\text { Odds } \\
1.27\end{array}$} & \multicolumn{2}{|c|}{$95 \% \mathrm{Cl}$} & \multirow{2}{*}{$\frac{p}{0.202}$} & \multirow{2}{*}{$\begin{array}{c}\text { Odds } \\
1.34\end{array}$} & \multicolumn{2}{|c|}{$95 \% \mathrm{Cl}$} & \multirow{2}{*}{$\begin{array}{l}\boldsymbol{p} \\
0.135\end{array}$} \\
\hline & & 1.02 & 1.98 & & & 0.88 & 1.84 & & & 0.91 & 1.95 & \\
\hline Log SuPAR & 2.42 & 1.27 & 4.59 & 0.007 & 1.42 & 0.66 & 3.08 & 0.371 & 1.39 & 0.61 & 3.14 & 0.435 \\
\hline Age & & & & & 0.99 & 0.95 & 1.02 & 0.447 & 0.98 & 0.94 & 1.02 & 0.294 \\
\hline gender (ref. male) & & & & & 1.18 & 0.61 & 2.28 & 0.624 & 1.32 & 0.65 & 2.68 & 0.447 \\
\hline $\mathrm{BMI}$ & & & & & 1.01 & 0.93 & 1.09 & 0.866 & 1.00 & 0.91 & 1.09 & 0.976 \\
\hline $\mathrm{SBP}^{\star}$ & & & & & 1.01 & 0.99 & 1.04 & 0.262 & 1.03 & 1.00 & 1.06 & 0.026 \\
\hline $\mathrm{HbA1c}$ & & & & & 0.93 & 0.65 & 1.33 & 0.704 & 0.99 & 0.68 & 1.43 & 0.950 \\
\hline $\log$ eGFR ${ }^{\star}$ & & & & & 0.10 & 0.03 & 0.32 & $<0.001$ & 0.12 & 0.03 & 0.46 & 0.002 \\
\hline duration & & & & & & & & & 0.99 & 0.95 & 1.04 & 0.729 \\
\hline $\mathrm{DBP}^{\star}$ & & & & & & & & & 0.96 & 0.93 & 1.00 & 0.071 \\
\hline $\mathrm{ALT}$ & & & & & & & & & 1.00 & 0.97 & 1.03 & 0.970 \\
\hline $\mathrm{TC}^{\star}$ & & & & & & & & & 0.99 & 0.97 & 1.00 & 0.093 \\
\hline TG & & & & & & & & & 1.00 & 1.00 & 1.01 & 0.279 \\
\hline $\mathrm{HDL}^{*}$ & & & & & & & & & 1.03 & 1.00 & 1.06 & 0.049 \\
\hline$U A^{\star}$ & & & & & & & & & 1.22 & 1.02 & 1.45 & 0.026 \\
\hline
\end{tabular}

Two-year ACR deterioration shown by logistic regression analysis based on whole DKD. Model 1: only log(PS + 1) and Log suPAR (crude model), Model 2: Model 1 with known factors (age, gender, BMI, SBP, HbA1c and log eGFR) ${ }^{23}$, Model 3: Model 2 with other factors (duration, DBP, ALT, TC (total cholesterol), TG (triglyceride), HDL (high-density lipoprotein), and UA (uric acid)). The $95 \%$ confidence interval $(95 \% \mathrm{Cl})$ is listed

Remaining variables after the stepwise method based on the Akaike's information criterion (AIC) in model 3 are depicted as *

The TPL-specific inhibitors 2-aza-tyrosine and L-meta-tyrosine reduced not only plasma and tissue PS levels, but also IS levels in the diabetic mouse model (Fig. 4b). PS is a metabolite of the phenol that is generated by TPL (EC 4.1.99.2) using tyrosine as a substrate. IS is generated by tryptophan indole lyase (TIL, EC 4.1.99.1) using tryptophan as a substrate 31,32 (Fig. 4a). TPL and TIL enzymes show high-sequence homology ${ }^{32}$. It is conceivable that TPL inhibitors also partially inhibit TIL, resulting in a simultaneous reduction in PS and IS concentrations. To clarify this, we examined the effect of TPL inhibitors on TIL activity ${ }^{33}$. The specific TIL inhibitor 2-amino-4-(benzimidazol-1-yl)butyric acid (homo-BZI-Ala) at $100 \mu \mathrm{M}$, significantly inhibited TIL (apotryptophanase) activity as reported ${ }^{33}$ (Fig. 4h). Concentrations of $1 \mathrm{mM} 2$-aza-tyrosine and L-meta-tyrosine partly, though significantly, inhibited TIL activity. These data suggest that 2-azatyrosine inhibits both TPL and TIL, resulting in a dual reduction in plasma PS and IS concentrations followed by the recovery of renal damage.

2-Aza-tyrosine shows low-microbiota taxa pressure in RF mice. To further investigate the effect of 2-aza-tyrosine on the gut microbiota, we performed microbial $16 \mathrm{~S}$ rRNA gene sequencing of feces. The $\alpha$-rarefaction (observed OTUs) (Fig. 5a), $\alpha$-diversity (Fig. 5b), and principal coordinates analyses using weighted UniFrac analysis (Fig. 5c) revealed that 2-aza-tyrosine did not cause a major shift in the major gut microbial composition. Next, we focused on the microbiome in which the number positively correlated with the plasma PS level in renal failure and was reduced by 2 -aza-tyrosine. At the order level, the major population of gut microbiota (Bacteroidales, Clostridales, and Lactobacillales) became more pronounced at $>90 \%$ and did not follow the criteria (Fig. 5d and Supplementary Fig. 10). However, two minor populations of microbial order (Coriobacteria and Erysipelotrichales) showed a significant increase in renal failure, and these increments were markedly decreased by 2 -aza-tyrosine (Fig. 5e and Supplementary Fig. 10). At the family level, Coriobacteriaceae and Erysipelotrichaceae were significantly increased in renal failure and this rise was rescued by 2 -aza-tyrosine (Fig. 5e and Supplementary Figs. 11 and 12). At the genus level, the composition of the 12 major genera was not altered in renal failure mice with or without 2-aza-tyrosine (Fig. 5e and Supplementary
Figs. 13-15). However, Adlercreutzia and unclassified Erysipelotrichaceae positively correlated with plasma PS concentration, suggesting that these two minor taxa might, in part, influence the change in the plasma PS level. TPL activity was found in the Enterobacteriaceae family (Citrobacter, Proteus, and Erwinia) ${ }^{26}$, but in this experiment, the relative abundance of Enterobacteriaceae was very small (less than $0.1 \%$ ) and no correlation was observed in the plasma PS concentration (Fig. 5e). We also found that, after 1 week of cessation of $\mathrm{L}$-m-tyrosine $(10 \mathrm{mg} / \mathrm{kg})$ administration in $\mathrm{db} / \mathrm{db}$ mice, the reduced plasma PS level recovered (Supplementary Fig. 16). These data suggest that TPL inhibitor did not significantly alter the major composition, but it reduced the PS production with a minor taxon modification.

Taken together, these data suggest that PS is a marker and a modifiable therapeutic target for DKD patients, especially those with microalbuminuria, and TPL inhibitor did not significantly alter the major composition, but it reduced the PS production with a minor taxon modification (summarized in Fig. 6).

\section{Discussion}

It has been reported that the gut-derived metabolite PS is markedly increased with the progression of renal failure ${ }^{34}$ and is also higher in hemodialysis patients ${ }^{35}$. However, the precise biological activity or toxicity has not been clarified. The cause of $\mathrm{DKD}$, includes irreversible (age, gender and duration of diabetes) and modifiable (hyperglycemia, hypertension and albuminuria) risk factors ${ }^{36}$. Intense multifactorial intervention targets glycemic control, blood pressure, including renin-angiotensin system blockade and dyslipidemia ${ }^{37}$. However, diabetic patients still have a poorer outcome ${ }^{38}$. The main pathophysiological changes in DKD kidney, include podocyte damage, thickening of the GBM, interstitial fibrosis, and hyalinosis of blood vessels ${ }^{39}$, as well as mitochondrial dysfunction in the podocytes ${ }^{16}$. We found that PS was toxic in podocytes and also reduced mitochondrial function. PS thickened the GBM and elicited inflammation with perivascular fibrosis. These multitoxicities of PS on the DKD kidney might lead to increased albuminuria. Therefore, DKD patients with a high-PS level should reduce the PS level to prevent the progression of albuminuria.

Although the limitations have been reported ${ }^{40}$, microalbuminuria is still a central target in the prevention and 
a

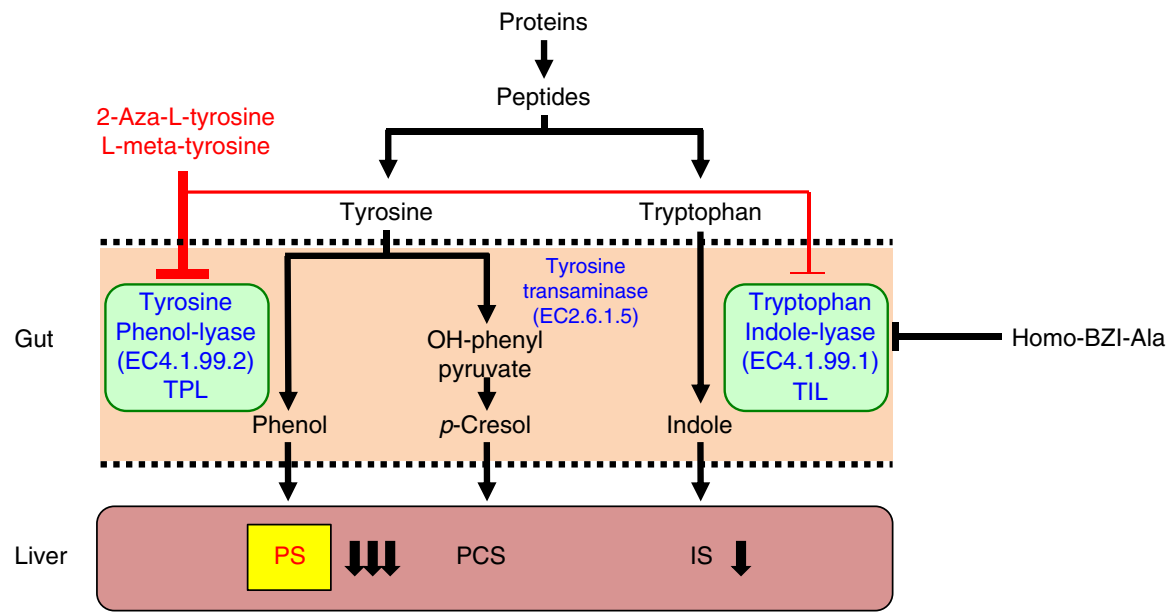

b

PS

PCS

IS

TMAO

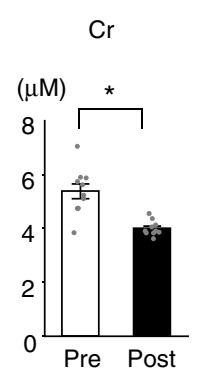

C


d

PS

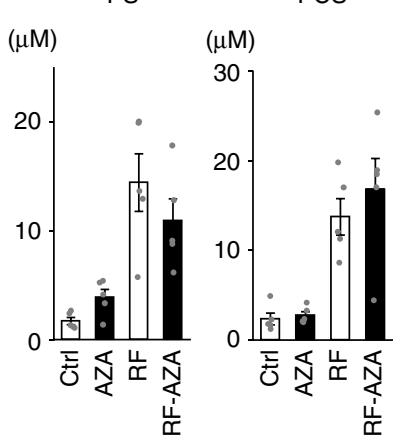

$\mathbf{f}$

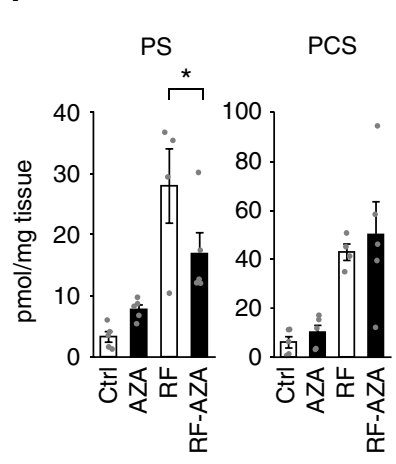

IS

TMAO e

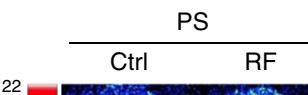

$\mathrm{Cr}$
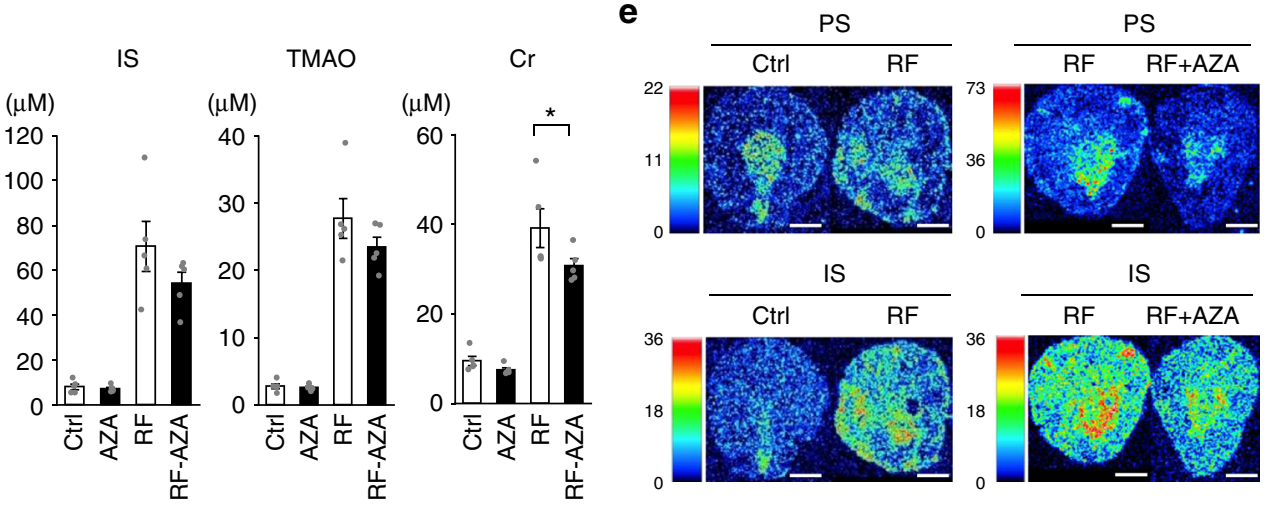

20.
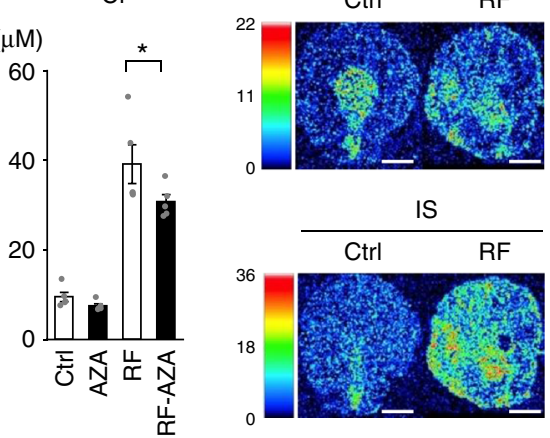

IS
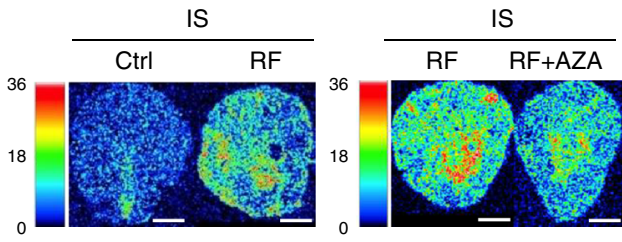

g
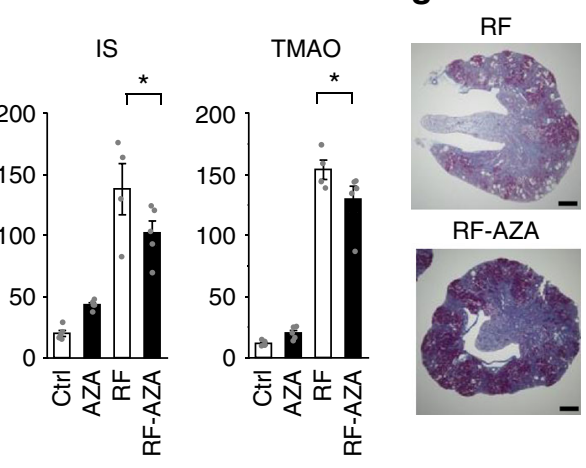

h

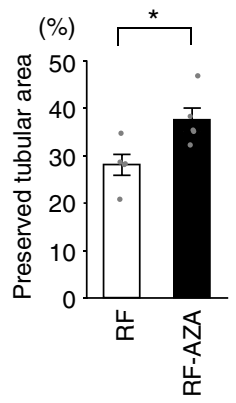

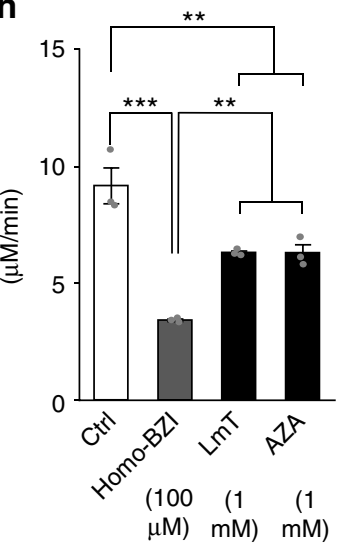

treatment of $\mathrm{DKD}^{37}$, and identifying which patients with microalbuminuria are most likely to develop more advanced kidney disease is important. We found that PS is related to the basal ACR and to the progression of albuminuria, suggesting the effectiveness of measuring PS in DKD patients with microalbuminuria. In addition, reducing circulating PS levels might also reduce the risk of DKD development (Fig. 6).

The circulating concentrations of uremic toxins is dependent on the variety of diet and time to draw blood ${ }^{41}$. To clarify the 
Fig. 4 TPL inhibitors lowered PS concentrations and reduced renal damage in mouse models of diabetic kidney disease and adenine-induced renal failure. a Scheme of gut microbiome-derived PS, PCS, and IS generation. Dietary tyrosine is converted to phenol by tyrosine phenol-lyase (TPL) and further modified to produce PS or PCS. Dietary tryptophan is first converted to indole by tryptophan indole-lyase (TIL), further converted and finally modified into IS. Specific inhibitors of TPL are 2-aza-tyrosine and L-meta-tyrosine, while homo-BZI-Ala is a specific inhibitor of TIL. b Plasma concentrations of PS, IS, $\mathrm{PCS}, \mathrm{TMAO}$, and $\mathrm{Cr}$ in $\mathrm{db} / \mathrm{db}$ mice before (Pre) and after treated with 2-aza-tyrosine (post, $10 \mathrm{mg} / \mathrm{kg}, n=10$ ). c Urinary albumin/creatinine ratio of $\mathrm{db} / \mathrm{db}$ mice with either no intervention (Ctrl) or treatment with the TPL inhibitor 2-aza-tyrosine (AZA). N=6 in each group. $\mathbf{d}$ Plasma concentrations of PS, IS, $\mathrm{PCS}, \mathrm{TMAO}$, and $\mathrm{Cr}$ in the adenine-induced renal failure mouse model treated with 2-aza-tyrosine $(5 \mathrm{mg} / \mathrm{kg})$ for 14 days. Four groups were measured: control mice (Ctrl), control mice treated with 2-aza-tyrosine (AZA), adenine-induced renal failure mice with no intervention (RF), and adenine-induced renal failure mice treated with 2-aza-tyrosine (RF-AZA). $n=5$ in each group. e Representative imaging mass spectrometry spectra of PS and IS in kidney sections from renal failure mice (RF) and renal failure mice administered 2-aza-tyrosine $(5 \mathrm{mg} / \mathrm{kg})(\mathrm{RF}+\mathrm{AZA})$. Mass spectrometry imaging shows the distribution of PS $\left([\mathrm{M}-\mathrm{H}]^{-}, \mathrm{m} / \mathrm{z} 172\right)$ and IS $\left([\mathrm{M}-\mathrm{H}]^{-}, \mathrm{m} / \mathrm{z} 212\right)$. Scale bar, $1000 \mu \mathrm{m}$. f Quantitative analysis of PS and IS tissue contents in mouse kidneys in RF $(n=4)$ and others $(n=5)$. $\mathbf{g}$ Morphometric analysis of the fractional cortical tubular area of Masson trichrome stained whole kidney images. $n=5$ in each group. Data are presented as the percentage of the total cortex $(n=5)$. Scale bar, $500 \mu \mathrm{m}$. $\mathbf{h}$ Enzymatic activity of TIL. TIL activity under control conditions (Ctrl), with TIL inhibitor homo-BZI-Ala $(100 \mu \mathrm{M})$ and a tyrosine phenol-lyase inhibitors (L-meta-tyrosine (LmT) or 2-aza-tyrosine (AZA), $1 \mathrm{mM}), n=3 .{ }^{*} p<0.05$ vs. control according to Paired $t$ test $(\mathbf{b})$, data are mean $\pm \mathrm{SEM}{ }^{*} p<0.05$ vs. control according to $t$ test (c, d, $\left.\mathbf{g}, \mathbf{f}\right)$ or Tukey-Kramer test $\left(\mathbf{h},{ }^{\star} p<0.05,{ }^{\star \star} p<0.01,{ }^{\star \star *} p<0.001\right)$. Source data are provided as a Source Data file

dietary effect of tyrosine on the PS concentration, we measured the PS levels in plasma collected serially over time in control (C57BL6) and diabetic (db/db) mice. After oral tyrosine administration, a greater increase in plasma PS level in diabetic mice occurred during the 4 -h study period and peaked at $2 \mathrm{~h}$ compared with that of control (Supplementary Fig. 17). In addition, the serum PS level was higher in diabetic mice. The restriction of dietary protein is recommended for reducing glomerular hyperfiltration and pro-inflammatory gene expression in both CKD and DKD patients ${ }^{42}$. Our data suggests another aspect that reducing tyrosine intake in diabetic patients might decrease PS production, thereby reducing albuminuria and being renoprotective.

Concerning eGFR, our analysis uses only two time points of eGFR and the median eGFR decline was -1.8 in 2 years. Since the change in eGFR was small, we will need another cohort with a long observation period to reach a clear conclusion on the relation between PS and eGFR. In addition, under the absent decline in GFR, there is no role for circulating factor suPAR. The difference in the sensitivity between PS and suPAR may not be an issue with suPAR but rather with the cohort.

2-Aza-tyrosine reduced both PS and IS in the diabetic model. It is well-known that the charcoal adsorbent AST-120 significantly reduces PS and $\mathrm{IS}^{35}$. IS accelerates the progression of $\mathrm{CKD}^{43}$, but the contribution on albuminuria was limited ${ }^{43}$. Administration of IS to renal failure rats declined renal function, but the proteinuria was not changed ${ }^{43}$ In diabetic patients with normal renal function, AST-120 reduced the progression of renal dysfunction without changing IS level ${ }^{44}$. 2-Aza tyrosine decreased both PS and IS, the contribution of PS to albuminuria in $\mathrm{db} / \mathrm{db}$ mice might be more potent than IS.

Our data showed that proteinuria was rapidly reduced in PSloaded animals and we found that PS has a podocyte mitochondrial toxicity. It was reported that podocyte-specific loss of PHB2, a mitochondrial inner membrane structural protein led to early onset of proteinuria in 3-weeks and death after 5 weeks ${ }^{45}$. On the other hand, in our previous experiment, mitochondrial DNA knockout mice generate renal damage after 7-9 months ${ }^{46}$. The difference in onset may be attributed to differences in the model. We found that the combination PS and suPAR improved the $c$-statistics and this phenomenon raised another possibility of another mechanism of podocyte injury, e.g., suPAR is binding to and activating av a3 integrins on podocytes, a process that leads to activation of small GTPase Rac-1 which in turns drives podocyte foot process motility and foot process effacement ${ }^{47}$.
The interference of gut microbiota may involve in the pathogenesis or development of diabetes ${ }^{48}$ and $\mathrm{CKD}^{49}$. However, little is known regarding $\mathrm{DKD}$. It was reported a significant disruption of the epithelial tight junction in different $\mathrm{CKD}$ models ${ }^{50}$. In HFD-induced obesity and diabetes mice, expression of the tight junction proteins zonula occludens-1 (ZO-1) and occludin decreased $^{51}$. We examined the epithelial tight junction in WT and transgenic rats and the expression levels of claudin I, occludin, and ZO-1 in the ileum and colon were unchanged (Supplementary Fig. 18).

We found that the microbial orders Coriobacteria and Erysipelotrichales increased in renal failure and decreased by 2 -azatyrosine. Coriobacteriaceae are gram-positive anaerobic bacteria and in our previous study, the Coriobacteriaceae family and Adlercreutzia were increased in renal failure mouse, but our intervention did not alter the increase ${ }^{52}$. It was reported that in CKD patients, the low-GFR group showed increased Adlercreutzia, which positively correlated with the TMAO level ${ }^{53}$. Erysipelotrichale are also gram-positive anaerobic bacteria and some types have been isolated from human feces and the bacteria encodes TPL ${ }^{54}$. Erysipelotrichales showed a decrease in Crohn disease patients ${ }^{55}$, but little has been clarified the relation to renal disease.

TPL has been found in a number of different strains of bacteria, most of which belong to the Enterobacteriaceae family, specifically to the genera Citrobacter, Proteus, and Erwinia ${ }^{26}$. However, the Enterobacteriales family and the Enterobacteriaceae family represent a minor population and little is known about the pathogenic contribution of such gut microbiota to human diseases. We also found that the microbial genus Adlercreutzia and Erysipelotrichaceae unclassified significantly correlated with a change in the PS levels. However, the TPL gene was not reported in the taxa according to the Genbank database search. Further experiments are needed to clarify the microbiome that produces phenol in renal failure.

The production of phenols and indoles by gut bacteria is associated with a variety of diseases in humans and animals. Phenols have been implicated as having a possible role in the causation of large bowel cancer ${ }^{56}$ and as causal factors in leukemia ${ }^{57}$. Elevated plasma levels of the phenol derivative 4-ethylphenylsulfate may promote anxiety-like behavior ${ }^{58}$. Chemical inhibition of TPL may also have a beneficial effect in preventing the phenol-induced disorders.

In conclusion, PS is not only an early diagnosis marker, but also a modifiable cause and therefore a target for the treatment of DKD. Chemical reduction of microbiota 
a

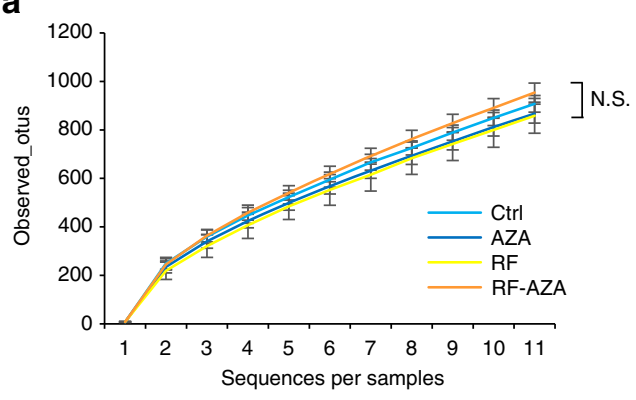

C

$\begin{array}{ll}\text { O Ctrl } & \text { ORF } \\ \text { O AZA } & \text { ORF-AZA }\end{array}$
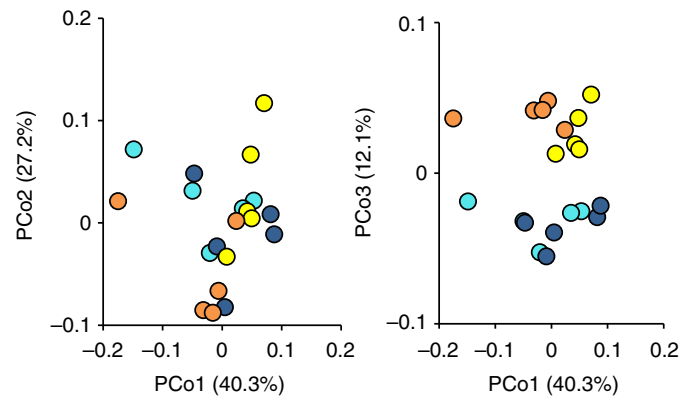

b
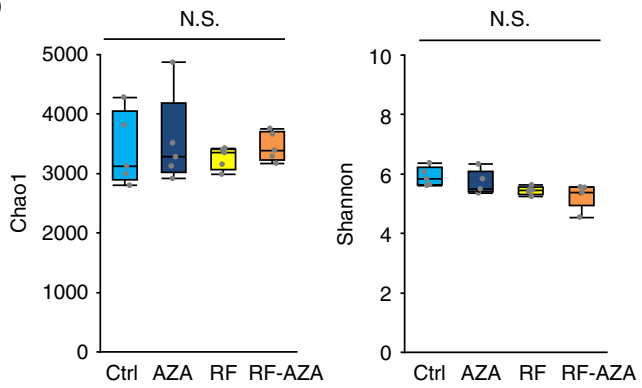

d

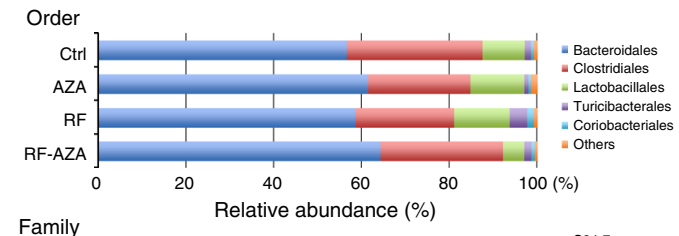

Family , Relative abundance (\%), is $24-7$
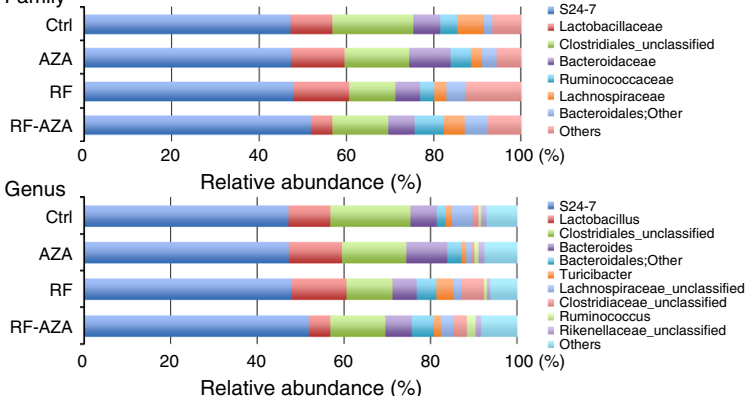

e
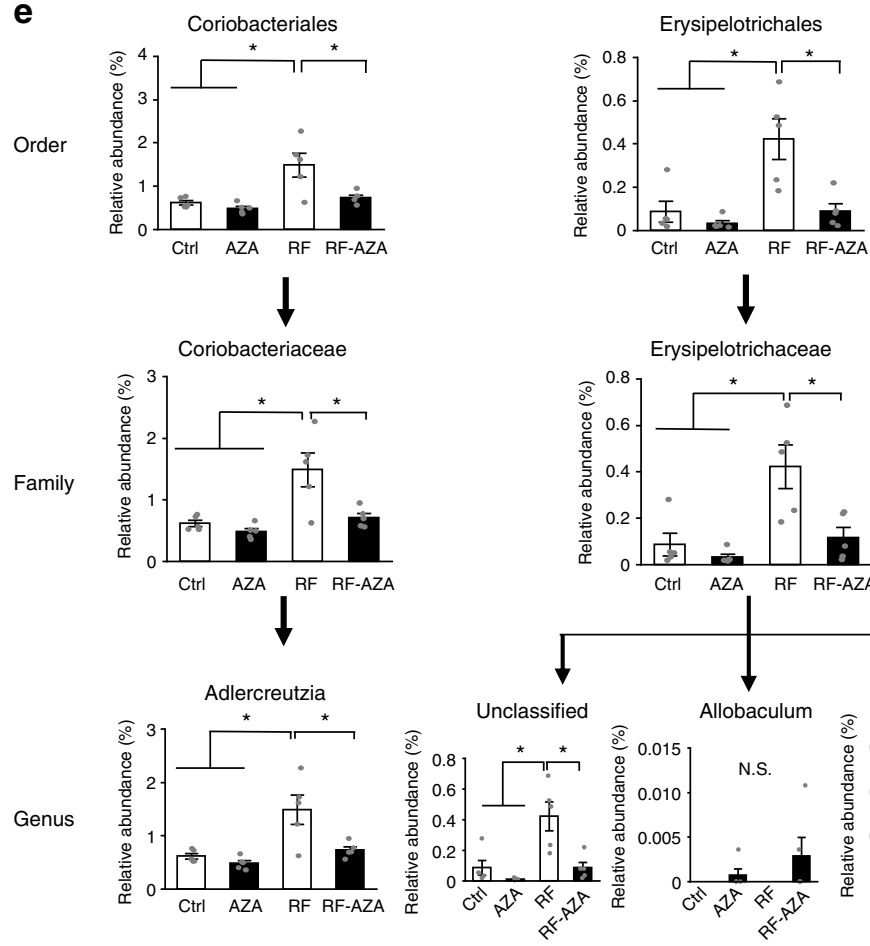

Erysipelotrichaceae
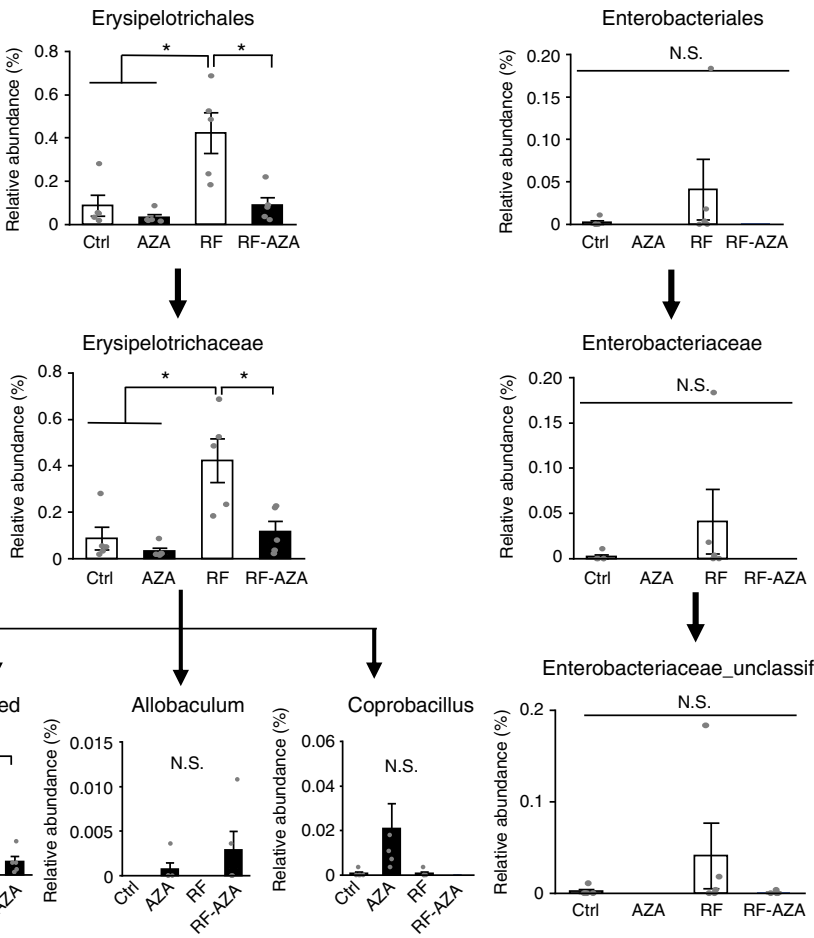

Enterobacteriaceae_unclassified

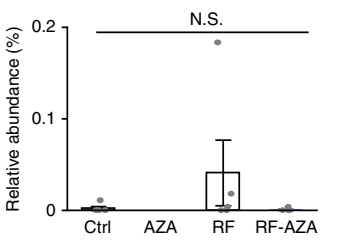

TPL should represent another aspect for developing drugs preventing DKD.

\section{Methods}

Materials. PS and ${ }^{13} \mathrm{C}_{6}$ labeled PS were synthesized by Sundia MediTech

(Shanghai, China) and ISOTEC (Sigma-Aldrich), respectively. 2-Aza-tyrosione ${ }^{27}$ and 2-amino-4-(benzimidazol-1-yl)butyric acid (Homo-BZI-Ala) ${ }^{33}$ were chemically synthesized. L-meta-tyrosine was purchased from Astatech (Bristol, PA). HUPEC ${ }^{13}$ were provided by Dr. Jeffrey B. Kopp (NIH, Bethesda). Human kidney proximal tubule cell line HK-2 (CRL2190) and Canine kidney cell line MDCKII (CRL2936) were purchased from ATCC.

Animal experiments. The animal protocols were approved by the Tohoku University Institutional Animal Care and Use Committee (2016-007-1, 2-16-008-3). 
Fig. 5 Effect of 2-aza-tyrosine (AZA) on the gut microbiome in adenine-induced renal failure mice. a Observed OTUs rarefaction analysis. OTU rarefaction curves of gut microbiome were used to estimate richness in the following groups: control mice (Ctrl); control mice treated with $5 \mathrm{mg} / \mathrm{kg} 2$-aza-tyrosine (AZA); adenine-induced renal failure mice (RF); and adenine-induced renal failure mice treated with $5 \mathrm{mg} / \mathrm{kg}$ 2-aza-tyrosine (RF-AZA). b OTU-based $\alpha$ diversity of each microbiome. No significant diversity was seen in any group by Chao1 (left) and Shannon (right) analyses. c Principal coordinates analysis of the microbiome profiles using weighted UniFrac. Scores are presented for the first principal component (PCo1) vs. both the second principal component (PCo2) and the third principal component (PCo3). d Relative abundance of microbiota based on the average number of each subgroup at order, family and genus levels. The major subgroups are indicated on the right. $\mathbf{e}$ The relative abundance of microbiota differed significantly among the four groups analyzed. The $y$-axis indicates the relative abundance of each microbe as a percentage. The proportional change in the minor groups correlated with renal failure and 2-aza-tyrosine treatment. The major microbial order (Bacteroidales, Clostridales, and Lactobacillales) were unchanged by either renal failure or 2-azatyrosine treatment. However, the minor populations (Coriobacteriales and Erysipelotrichales) were significantly changed in the correlation with the PS concentration by renal failure and 2-aza-tyrosine treatment. $n=5$ in each group. Data were mean \pm SEM. Statistical analysis was performed by the Tukey-Kramer test, followed by FDR correction of $p$ values. ${ }^{*} p_{\mathrm{FDR}}<0.1$ was treated as statistically significant. The following groups were tested: control group (Ctrl); control group administered 2-aza-tyrosine (AZA); the renal failure group (RF); and the renal failure group administered 2-aza-tyrosine (RFAZA). Metagenome Source data are provided as in a Source Data file

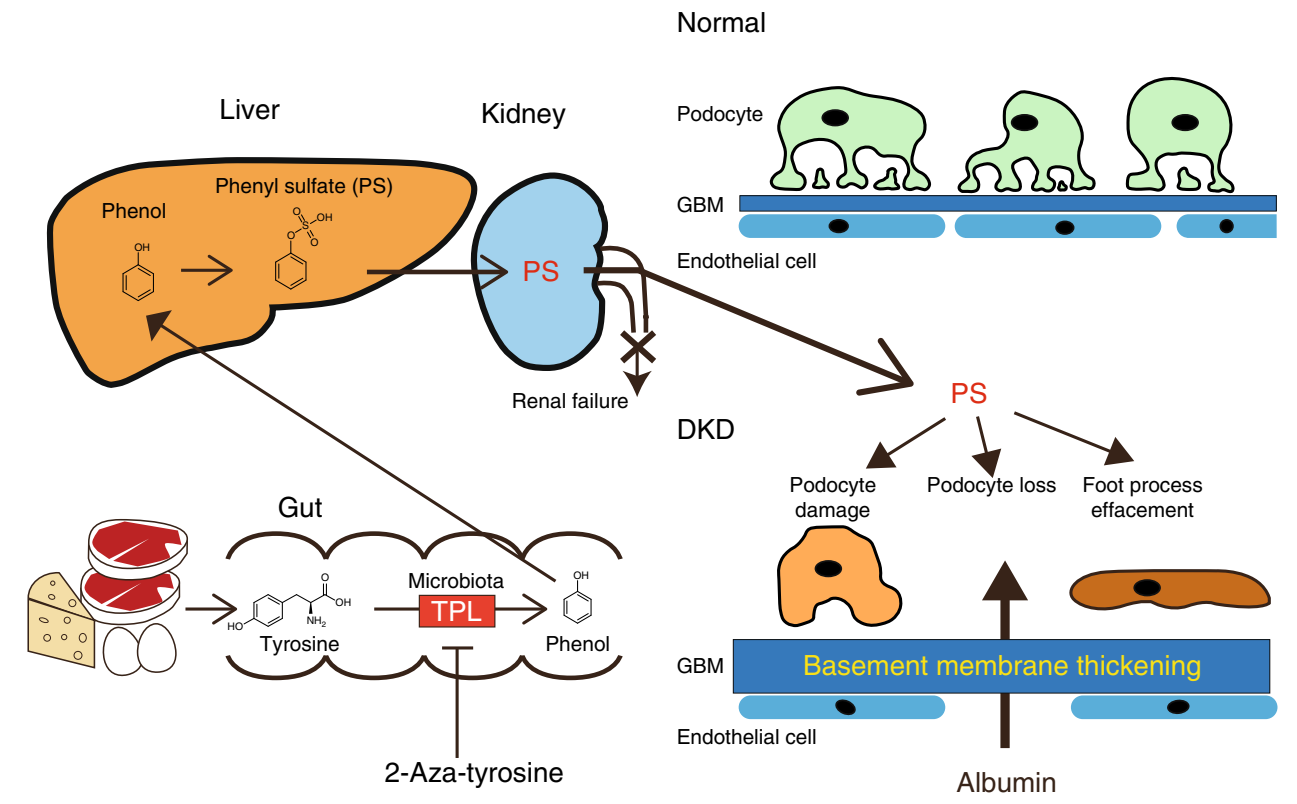

Fig. 6 A schematic model of the generation and toxicity of PS. In gut microbiota, TPL converts tyrosine to phenol and ammonia. PS is also generated in the liver. PS accumulates in plasma as a metabolite and has deleterious effects on the vasculature and kidneys. In diabetic kidney disease, PS damages podocytes, accelerates GBM thickening, and induces proteinuria. Treatment with TPL inhibitors reduces plasma PS levels and prevents the progression of renal failure in animal models

This study was conducted in accordance with the Guide for the Care and Use of Laboratory. SLCO4C1-Tg rats ${ }^{6}$ were bred at Tohoku University. C57BL6 mice, $\mathrm{db} /$ $\mathrm{db}$ mice and KKAy mice were obtained from CLEA Japan (Tokyo, Japan). Diabetes was induced in 8 -week-old SLCO4C1-Tg rats ${ }^{6}$ by an intraperitoneal injection of STZ $(50 \mathrm{mg} / \mathrm{kg})$ dissolved in a $0.1 \mathrm{M}$ citrate-phosphate buffer $(\mathrm{pH} 4.2)^{59}$. We estimated drug intake (PS, $50 \mathrm{mg} / \mathrm{kg} /$ day; 2 -aza, $5 \mathrm{mg} / \mathrm{kg} /$ day; and L-meta-tyrosine, $10 \mathrm{mg} / \mathrm{kg} /$ day) by measuring the amount of water intake. For the renal failure model, 8 -week-old C57BL/6N Jcl mice were fed a diet containing $0.2 \% \mathrm{wt} / \mathrm{wt}$ adenine for 6 weeks to induce tubular injury ${ }^{29}$, each group was further divided and 2 -aza-tyrosine $(10 \mathrm{mg} / \mathrm{kg})$ was orally administrated for 2 weeks with normal chow. The urinary protein level was determined by enzyme-linked immunosorbent assay (ELISA) (Nephrat II, Exocell Inc., Philadelphia, PA). Plasma was collected from the experimental rats and the glucose, insulin, creatinine and BUN concentrations were measured according to the kit instructions (Wako Pure Chemical, Osaka, Japan).

Histological examination. Kidney was fixed in 10\% neutral buffered formalin and embedded in paraffin. Kidney sections were stained with periodic acid-Schiff (PAS), Masson's trichrome, and Sirius red. For morphometric analysis of the glomeruli, PAS-stained sections were used for histomorphometric analysis. The whole-glomerular tuft volume was measured according to the reported method ${ }^{60}$. The area was quantified by measurement at $\times 200$ magnification in 50 random nonoverlapping fields in each group $(n=4)$ using Image J (Figs. 1b and 2c). In SLCO4C1 TG rats, the preserved tubular area and fibrotic area (Sirius Red positive area) were quantified by measurement at $\times 20$ magnification in whole renal cortex area in the field using Image J software (NIH, Bethesda, MD). In mice, the glomerular sclerosis area was measured using PAS-stained sections by Image J software, as reported ${ }^{19}$. In each analysis, 50 sections were examined from 3 animals in each examination. The preserved tubular area was also quantified by measurement at $\times 20$ magnification in the whole renal cortex area in the field using Image J. The fibrotic area was quantified by measurement at $\times 200$ magnification in 20 random nonoverlapping fields using Image ${ }^{61}$. Electron microscopic analysis was performed as previously reported ${ }^{17}$. The mesangial matrix score was determined in at least 450 glomeruli from a total of 10 mice. Two pathologists, blind to the treatment of the individual rats or mice, performed the evaluation.

Untargeted metabolomics. Plasma samples were taken from STZ-treated wild type rats: $\mathrm{d} 7(n=6), \mathrm{d} 63(n=3)$, and $\mathrm{d} 119(n=3)$. Plasma samples were also taken from STZ-treated transgenic rats: $\mathrm{d} 63(n=3)$ and $\mathrm{d} 119(n=4)$. Plasma samples were deproteinized by adding equal volumes of acetonitrile, then vortexed and centrifuged at $15,000 \times g$ for $5 \mathrm{~min}$ at room temperature and the supernatants were analyzed. UHPLC-QTOF/MS analysis was performed on an Acquit Ultra Performance LC I-class system (Waters Corp. Milford, MA) connected to a Waters Synapt G2-Si QTOF MS fitted with an electrospray ionization (ESI) source operated in the negative ion mode. Samples were separated using a Waters Acquity UPLC BEH Amide Column $(1.7 \mu \mathrm{m}, 2.1 \times 150 \mathrm{~mm})$ kept at $45^{\circ} \mathrm{C}$ and a flow rate of $0.4 \mathrm{~mL} / \mathrm{min}$. Acetonitrile $(10 \mathrm{mM}$ ammonium bicarbonate $(95: 5, \mathrm{v} / \mathrm{v})$ was used as mobile phase A and acetonitrile (10 mM ammonium bicarbonate $(5: 95, \mathrm{v} / \mathrm{v})$ was used as mobile phase B. The gradient was applied as follows: $1 \% \mathrm{~B}$ at $0-0.1 \mathrm{~min}$, 
$1-70 \% \mathrm{~B}$ at $0.1-6.0 \mathrm{~min}, 70-1.0 \% \mathrm{~B}$ at $6.0-6.5 \mathrm{~min}$, and $1 \% \mathrm{~B}$ at $6.5-10.0 \mathrm{~min}$. All data were processed using ProgenesisQI software (Nonlinear Dynamics, Newcastle, UK) for peak picking, alignment, and normalization to produce peak intensities for retention time $\left(t_{\mathrm{R}}\right)$ and $\mathrm{m} / z$ data pairs. Features were identified from the Chemspider DB, Human Metabolome Database (HMDB) and Lipidmaps using the precursor and fragment ion spectra obtained by MS. The intensities of the identified features were imported to EZinfo software (Waters) for multivariate analysis, and their relative quantities were evaluated by PCA, PLS-DA, and OPLS-DA.

Quantification of PS, PCS, IS, and TMAO by LC/MS/MS methods. PS, PCS, IS, and TMAO levels in animal plasma were measured as we recently reported ${ }^{8}$. Briefly, deuterated internal standards were used for all analytes and a TSQ Quantum Ultra triple quadrupole mass spectrometer (Thermo Fisher Scientific, Waltham, MA) equipped with a heated ESI source system was used. The mass spectrometer was operated in both positive and negative ionization modes. Samples were analyzed in single reaction monitoring mode by monitoring the ion transitions: $m / z$ 173.0->93.3 for PS. Data were acquired and analyzed using Xcalibur ${ }^{\text {mm }}$ software (version 2.1, Thermo Scientific). For the human samples, the PS level in fresh-frozen plasma was measured by another method to help clinicians measuring PS by LSI Medience Co. (Tokyo, Japan). Briefly, LC-MS/MS data sets were acquired with a liquid chromatograph Nexera X2 LC0AD equipped with an ESI and triple quadrupole mass spectrometer 8050 (Shimadzu, Japan). The mass spectrometer was operated in the negative mode. The main parameters of the mass spectrometer are summarized in Supplementary Fig. 19. Data acquisition and analysis were performed using Shimadzu Lab Solutions version 5.89. The correlation of each measurement was confirmed with the correlation $>0.99269$ (Supplementary Fig. 19). The plasma levels of suPAR were measured by ELISA using the Quantikine Human suPAR Immunoassay kit (R\&D Systems, Minneapolis, $\mathrm{MN}$ ) according to the manufacturer's instructions.

Uptake experiment. Madin-Darby Canine Kidney (MDCK) II cells were stably transduced with SLCO4C1 or an empty vector and uptake experiments were performed as previously reported ${ }^{9}$. Uptake in HK-2 cells was initiated by adding $50 \mu \mathrm{M}$ PS in the absence or presence of $100 \mu \mathrm{M}$ ritonavir, a SLCO4C1 inhibitor. Uptake was terminated after a $10 \mathrm{~min}$ incubation. The concentration of PS in the cells was measured using LC/MS/MS. The uptake was calculated by dividing the uptake amount by the protein content of the cells.

Cell toxicity assay. Differentiated HUPEC ${ }^{13}$ were cultured in RPMI1640 supplemented with $2 \%$ FBS and plated at $0.5 \times 10^{4}$ cells $/ 0.5 \mathrm{~mL}$ assay medium per well in 96-well collagen-coated culture plates and modified with the relevant culture condition, as reported ${ }^{14}$. After each compound was applied at the final concentration indicated, differentiated podocytes were cultured for $72 \mathrm{~h}$ and cell viability was measured by Cell Count Regent SF (Nacalai Tesque, Kyoto, Japan) as previously reported ${ }^{17}$. To measure the glutathione levels, a CellTiterGloLuminescent Cell Viability Assay kit and a GSH/GSSG-Glo Assay kit were used (Promega, Madison, WI).

Mitochondrial function measurement. Bioenergetic analysis of human cultured podocytes was carried out as previously described ${ }^{17}$. Briefly, the OCR and $\mathrm{pH}$ gradient (ECAR) of the fibroblasts from the Leigh patients or normal volunteers were measured using Seahorse XF 24 (Seahorse Bioscience). Cells were cultured in assay medium (70 mM sucrose, $220 \mathrm{mM}$ mannitol, $10 \mathrm{mM} \mathrm{KH}_{2} \mathrm{PO}_{4}, 5 \mathrm{mM} \mathrm{mgCl}_{2}$, $2 \mathrm{mM}$ HEPES, $1.0 \mathrm{mM}$ EGTA and $0.2 \%(\mathrm{w} / \mathrm{v})$ fatty acid-free bovine serum albumin (BSA), $\mathrm{pH}$ 7.2) without $\mathrm{CO}_{2}$ for $60 \mathrm{~min}$. After equilibration, cells were measured in the three respiration states followed by injections of three inhibitors of oxidative phosphorylation.

Recruitment and measurement of the U-CARE cohort study. The Urinary biomarker for continuous and rapid progression of diabetic nephropathy (UCARE) study ${ }^{20}$ is a multicenter, observatory clinical study that planned to investigate the urinary biomarkers in diabetic nephropathy (UMIN 00011525).

For diabetic nephropathy patient cohort study, we recruited 362 patients (UCARE, registered UMIN 00011525). The analysis was approved by both the Tohoku University Ethics committee (2017-1-870) and Okayama University Ethics committee (1702-026). The collection of human samples was performed in accordance to the Tohoku University (2017-1-870) and after obtaining written informed consent adopted by the Okayama University (UMIN 00011525 and 1702026). The study design and baseline characteristics were as follows: eligible participants were between 20 and 80 years of age with type 1 and type 2 diabetes and excluded people with liver cirrhosis and a new onset of malignancies within 5 years. Among 777 patients enrolled in 2012, 564 participants were eligible for enrollment in this study. An included criterion was that serum samples stocked in 2014 and clinical data between 2014 and 2016 would be available. Among the 564 participants, 362 patients with full data were selected. The phlebotomy protocol was as follows. Although blood at random visits could clot at room temperature for between $30 \mathrm{~min}$ and $2 \mathrm{~h}$, samples were centrifuged, frozen at $-80^{\circ} \mathrm{C}$ and shipped on dry ice to the Okayama University Hospital, where the samples were aliquoted and frozen at $-80^{\circ} \mathrm{C}$. Laboratory processing was performed at each enrolled hospital. Estimated GFR (eGFR) was calculated according to gender, age and the concentration of serum $\mathrm{Cr}$ according the Japanese equation Chronic Kidney Disease Epidemiology Collaboration (CKD-EPI) ${ }^{62}$. CKD-EPI is multiplied by a Japanese coefficient of 0.813 ; eGFR $=0.813 \times 141 \times \min (\mathrm{SCr} / \kappa, 1)$ a $\max$ $(\mathrm{SCr} / \kappa, 1)^{-1.209} \times 0.993^{\mathrm{Age}} \times 1.018$ [if female] $\alpha$, where $\mathrm{SCr}$ is serum creatinine, $\kappa$ is 0.7 for females and 0.9 for males, $\alpha$ is 0.329 for females and 0.411 for males, min indicates the minimum of $\mathrm{SCr} /$ or 1 , and max indicates the maximum of $\mathrm{SCr} /$ or 1 . Several baseline variables were measured as confounding factors. For BMI, some participants did not have information on BMI at baseline. To keep the sample size we imputed an average of BMI measured 1 year before baseline and 1 year after baseline.

Statistical analysis of clinical data in DKD patients. Parametric factors are presented as means and standard deviations or as medians and interquartile ranges. Nonparametric factors are presented as proportions by the result of Shapiro-Wilk test. As a result, skewed variables (eGFR, ACR, PS, and suPAR) were logarithmically transformed to improve normality prior to analysis. Since some PS values in the U-CARE study were calculated as 0 according to the measurement limit, we added 1 to calculate log transformed values. The correlation between the plasma PS level and various factors was calculated using the Spearman rank-order correlation.

To perform multiple regression analysis, we built 3 models as an independent variable examined by variance inflation factor $(\mathrm{VIF})<10$, and the plasma PS level and ACR were used as objective variables. Model 1 is the crude model. Model 2 is adjusted by known factors: age, gender, BMI, SBP, HbAlc, and log (eGFR) $)^{23}$. Model 3 is the full model, adjusted by model 2 plus other clinical factors in Supplementary Table 4 (DBP, ALT, TC, TG, HDL, and UA). Since blood sampling did not involve fasting and was strongly related to $\mathrm{HbAlc}$, we did not adjust serum glucose concentration in the following analyses. A logistic regression analysis was also used to identify the factors independently associated with the development of 2 -year ACR deterioration. We defined the increased ACR cases as follows ${ }^{63,64}$ : normoalbuminuric at baseline: a case that progressed from normoalbuminuria to microalbuminuria or macroalbuminuria. Microalbuminuric at baseline: a case that progressed from microalbuminuria to macroalbuminuria or a case with ACR that doubled or more. Macroalbuminuric at baseline: a case with ACR that doubled or more. To identify the factors independently associated with the development of 2year ACR, the same 3 multiple logistic regression models were used. Model 1: only $\log (\mathrm{PS}+1)$ and $\log ($ SuPAR $)$ (crude model). Model 2: Model 1 with known factors (age, gender, BMI, SBP, HbA1c, and $\log (\mathrm{eGFR}))^{23}$. Model 3: Model 2 with other factors (duration, DBP, ALT, TC, TG, HDL, and UA). Results are presented as odds ratios (ORs) with 95\% CIs. ORs for all continuous variables were computed for each standard deviation (SD) change. ${ }^{*} p<0.05$ was set as statistically significant. The stepwise method was also performed based on the Akaike's information criterion (AIC).

Microbiome analysis. The genomic DNA of gut microbiota was extracted from murine feces. The 16S rRNA genes in the DNA samples were analyzed using a MiSeq sequencer (Illumina, San Diego, CA ${ }^{29}$. Microbial genomic DNA was extracted using a phenol-chloroform standard protocol, with vigorous shaking with $0.1 \mathrm{~mm}$ zirconia/silica beads. The V1-V2 region of the $16 \mathrm{~S}$ rRNA genes was amplified from the extracted DNA using the bacterial universal primer set Rd1 SP sequence-27Fmod (5'-ACACTCTTTCCCTACACGACGCTCTTCCGATCT-A GRGTTTGATYMTGGCTCAG-3') and Rd2 SP sequence-338R (5'-GTGACTGG AGTTCAGACGTGTGCTCTTCCGATCT-TGCTGCCTCCCGTAGGAGT-3' ${ }^{\prime}{ }^{65}$. Polymerase chain reaction (PCR) was performed with Tks Gflex DNA Polymerase (Takara Bio Inc.) and amplification proceeded with one denaturation step at $94{ }^{\circ} \mathrm{C}$ for $1 \mathrm{~min}$, followed by 20 cycles of $98^{\circ} \mathrm{C}$ for $10 \mathrm{~s}, 55^{\circ} \mathrm{C}$ for $15 \mathrm{~s}$ and $68^{\circ} \mathrm{C}$ for $30 \mathrm{~s}$, with a final extension step at $68^{\circ} \mathrm{C}$ for $3 \mathrm{~min}$. The PCR products were purified using Agencourt AMPure XP (Beckman Coulter Inc.) and further amplified using a forward primer (5'-AATGATACGGCGACCACCGAGATCTACAC-NNNNNNN $\mathrm{N}$-ACACTCTTTCCCTACACGACGC-3') containing the P5 sequence, a unique 8 bp barcode sequence for each sample (indicated in N), and Rd1 SP sequence and a reverse primer (5'-CAAGCAGAAGACGGCATACGAGAT-NNNNNNN

N-GTGACTGGAGTTCAGACGTGTG-3') containing the P7 sequence, a unique 8-bp barcode sequence for each sample (depicted as $\mathrm{N}$ ), and $\mathrm{Rd} 2 \mathrm{SP}$ sequence. After purification using Agencourt AMPure XP (Beckman-Courter), mixed samples were prepared by pooling approximately equal amounts of PCR amplicons from each sample ${ }^{29}$. Finally, MiSeq sequencing was performed according to the manufacturer's instructions. In this study, $2 \times 300 \mathrm{bp}$ paired-end sequencing was employed. As a result, 687,826 paired-end reads were obtained. To assemble the reads, fast length adjustment of short reads (v1.2.11) ${ }^{66}$ was used. 615,077 pairedend reads were assembled. Assembled reads with an average $Q$ value $<25$ were filtered out using in-house script. The number of reads per sample was from 27,828 to 35,423 . From each sample, 27,828 filter-passed reads were randomly selected and used for further analysis. Reads were then processed using quantitative insights into microbial ecology (QIIME) (v1.9.1) pipeline ${ }^{67}$. Sequences were clustered into operational taxonomic units (OTUs) using $97 \%$ sequence similarity and OTUs were assigned to taxonomy using the uclust method.

In microbiome analysis, $P_{\mathrm{FDR}}$ was calculated using the Benjamini and Hochberg false-discovery rate method $(\alpha=0.05)$ to adjust for independent multiple comparisons $^{68}$. FDR calculation used the p.adjust function of the stats package of 
the $R$ statistical language, version 3.4 .2 (http://www.r-project.org/) ${ }^{69} .{ }^{\star} P_{\mathrm{FDR}}<0.1$ was treated as statistically significant. JMP Pro software version 12 (SAS Institute Inc., Cary, NC) was used for statistical analysis.

TIL (apotryptophanase) activity measurement. The assay was carried out in a cuvette ( $1 \mathrm{~cm}$ optical path length) in a BioSpectrometer Kinetic (Eppendorf, Hauppauge, NY) at $37^{\circ} \mathrm{C}$ as previously reported ${ }^{70}$, but with some modification. The reaction mixture contained $0.005 \%$ BSA (Sigma), $1 \mathrm{mM}$ reduced glutathione (Sigma), $100 \mu \mathrm{M}$ pyridoxal phosphate (Sigma), $100 \mu \mathrm{M}$ NADH (Sigma), $3 \mathrm{U} / \mathrm{mL}$ lactate dehydrogenase (Sigma) and apotryptophanase $(10 \mathrm{U} / \mathrm{mL}$, Sigma) in $0.15 \mathrm{M}$ potassium phosphate buffer ( $\mathrm{pH} 8.0$ ). Following a $3 \mathrm{~min}$ incubation with inhibitor, the enzymatic reaction was initiated by adding L-tryptophan $(300 \mu \mathrm{M}$ final, Sigma). The decrease in optical density at $340 \mathrm{~nm}$ was monitored for $5 \mathrm{~min}$ at $5 \mathrm{~s}$ intervals and used for calculating the TIL reaction velocity. The inhibitory effect of TPL inhibitors (2-aza-tyrosine and L-meta-tyrosine) on TIL enzymatic activity was measured. Homo-BZI-Ala was used as a positive control of TIL inhibitor.

Mass spectrometry imaging of kidney sections. MSI of kidney sections was performed as previously reported ${ }^{30}$. Briefly, kidney tissues were sectioned to $8 \mu \mathrm{m}$ in thickness with a cryostat and thaw-mounted onto indium tin oxide-coated glass slides. Using a $0.3 \mathrm{~mm}$ caliber nozzle airbrush (Procon boy FWA Platinum; Mr. Hobby), 9-AA $(750 \mu \mathrm{L}, 4.5 \mathrm{mg} / \mathrm{mL}$ in methanol) was sprayed on the slide. MALDIMSI analysis was performed using iMScope (Shimadzu, Japan). Mass spectra of the designated areas on a specimen photographed before matrix application were acquired in the negative ion mode. Mass spectra were acquired under the condition of a laser frequency and scanning mass ranging from $\mathrm{m} / \mathrm{z} 200$ to $\mathrm{m} / z 220$ and $\mathrm{m} / \mathrm{z} 170$ to $m / z$ 189. The spatial interval of data points was $50 \mu \mathrm{m}$, giving 10,120 data points in total for each section. The data collected through the microscopic system were digitally processed using Imaging MS Solution analysis software (Shimadzu).

Quantitative PCR analysis. Whole kidneys were homogenized in TRIzol reagent (Invitrogen) and extracted according to the manufacturer's directions. The cDNA synthesis was performed using a transcri ptor first strand cDNA synthesis kit (Roche). Primers purchased from Applied Biosystems are shown in Supplementary Table 10. The sequences of the primers and probes are certificated by the company, but not open based on the company policy.

Western blot. Western blotting of ileum and colon were performed as previously described $^{61}$. The primary antibodies against claudin 1 (1:1000 dilution, ab15098), occludin (1:1000 dilution, ab31721), and ZO-1 (1:1000 dilution, abG041) were purchased from Abcam.

Statistical analysis. The data are expressed as means \pm SEM or SD. ${ }^{*} p<0.05$ ${ }^{* *} p<0.01$, and ${ }^{* * *} p<0.001$ were considered statistically significant. JMP Pro software version 12 (SAS Institute., Cary, NC) was used for the statistical analyses.

Reporting summary. Further information on research design is available in the Nature Research Reporting Summary linked to this article.

\section{Data availability}

The data that support the findings of this study are available from the corresponding author upon reasonable request. The source data underlying Figs. 1a, b, 1g, 1j, 2a, b, 2d-g, 2i, j, 4b-d, 4f-h, 5a-e, and Supplementary Figs. 1, 3-7, 9, 16-19, Supplementary Tables 2 and 3 are provided as a Source Data file. Untargeted metabolome analysis underlying Fig. 1c-f, Supplementary Fig. 2a-d and Supplementary Table 1 are provided in a file named Supplementary Data 1. The microbiome data were deposited in the DDBJ database (https://trace.ddbj.nig.ac.jp/DRASearch/) under accession number DRA006253. A Reporting Summary for this Article is available as a Supplementary Information file.

Received: 26 September 2018 Accepted: 28 March 2019

Published online: 23 April 2019

\section{References}

1. Ritz, E. \& Orth, S. R. Nephropathy in patients with type 2 diabetes mellitus. $N$. Engl. J. Med. 341, 1127-1133 (1999).

2. Fineberg, D., Jandeleit-Dahm, K. A. \& Cooper, M. E. Diabetic nephropathy: diagnosis and treatment. Nat. Rev. Endocrinol. 9, 713-723 (2013).

3. Pavkov, M. E. et al. Early renal function decline in type 2 diabetes. Clin. J. Am. Soc. Nephrol. 7, 78-84 (2012).

4. Mikkaichi, T. et al. Isolation and characterization of a digoxin transporter and its rat homologue expressed in the kidney. Proc. Natl Acad. Sci. USA 101, 3569-3574 (2004).
5. Menni, C. et al. Biomarkers for type 2 diabetes and impaired fasting glucose using a nontargeted metabolomics approach. Diabetes 62, 4270-4276 (2013).

6. Toyohara, T. et al. SLCO4C1 transporter eliminates uremic toxins and attenuates hypertension and renal inflammation. J. Am. Soc. Nephrol. 20, 2546-2555 (2009).

7. Wiklund, S. et al. Visualization of GC/TOF-MS-based metabolomics data for identification of biochemically interesting compounds using OPLS class models. Anal. Chem. 80, 115-122 (2008).

8. Kanemitsu, Y. et al. Simultaneous quantitative analysis of uremic toxins by LC-MS/MS with a reversed-phase/cation-exchange/anion-exchange tri-modal mixed-mode column. J. Chromatogr. B Anal. Technol. Biomed. Life Sci. 10681069, 1-8 (2017).

9. Sato, T., Mishima, E., Mano, N., Abe, T. \& Yamaguchi, H. Potential drug interactions mediated by renal organic anion transporter OATP4C1. J. Pharmacol. Exp. Ther. 362, 271-277 (2017).

10. Mishima, E. et al. Evaluation of the impact of gut microbiota on uremic solute accumulation by a CE-TOFMS-based metabolomics approach. Kidney Int. 92, 634-645 (2017).

11. Brinkkoetter, P. T., Ising, C. \& Benzing, T. The role of the podocyte in albumin filtration. Nat. Rev. Nephrol. 9, 328-336 (2013).

12. Gallo, L. A. et al. Once daily administration of the SGLT2 inhibitor, empagliflozin, attenuates markers of renal fibrosis without improving albuminuria in diabetic db/db mice. Sci. Rep. 6, 26428 (2016).

13. Sakairi, T. et al. Conditionally immortalized human podocyte cell lines established from urine. Am. J. Physiol. Ren. Physiol. 298, F557-F567 (2010).

14. Edamatsu, T., Fujieda, A. \& Itoh, Y. Phenyl sulfate, indoxyl sulfate and pcresyl sulfate decrease glutathione level to render cells vulnerable to oxidative stress in renal tubular cells. PloS ONE 13, e0193342 (2018).

15. Itoh, Y., Ezawa, A., Kikuchi, K., Tsuruta, Y. \& Niwa, T. Protein-bound uremic toxins in hemodialysis patients measured by liquid chromatography/tandem mass spectrometry and their effects on endothelial ROS production. Anal. Bioanal. Chem. 403, 1841-1850 (2012).

16. Che, R., Yuan, Y., Huang, S. \& Zhang, A. Mitochondrial dysfunction in the pathophysiology of renal diseases. Am. J. Physiol. Renal Physiol. 306, F367-F378 (2014).

17. Matsuhashi, T. et al. Mitochonic acid 5 (MA-5) facilitates ATP synthase oligomerization and cell survival in various mitochondrial diseases. EBioMedicine 20, 27-38 (2017).

18. Jheng, H. F., et al. Dietary low-fat soy milk powder retards diabetic nephropathy progression via inhibition of renal fibrosis and renal inflammation. Mol. Nutr. Food Res. 61, https://doi.org/10.1002/ mnfr.201600461 (2017)

19. Oe, Y. et al. Coagulation factor Xa and protease-activated receptor 2 as novel therapeutic targets for diabetic nephropathy. Arterioscler. Thromb. Vasc. Biol. 36, 1525-1533 (2016).

20. Mise, K. et al. Identification of novel urinary biomarkers for predicting renal prognosis in patients with type 2 diabetes by glycan profiling in a Multicenter Prospective Cohort Study: U-CARE Study 1. Diabetes Care 41, 1765-1775 (2018).

21. Hayek, S. S. et al. Soluble urokinase receptor and chronic kidney disease. $N$. Engl. J. Med. 373, 1916-1925 (2015).

22. Guthoff, M., et al. Soluble urokinase receptor (suPAR) predicts microalbuminuria in patients at risk for type 2 diabetes mellitus. Sci. Rep. 7, 40627 (2017)

23. Hoshino, J. et al. A new pathological scoring system by the Japanese classification to predict renal outcome in diabetic nephropathy. PloS ONE 13, e0190923 (2018).

24. Parving, H. H. et al. Prevalence and risk factors for microalbuminuria in a referred cohort of type II diabetic patients: a global perspective. Kidney Int. 69 2057-2063 (2006).

25. Wang, Z. et al. Non-lethal inhibition of gut microbial trimethylamine production for the treatment of atherosclerosis. Cell 163, 1585-1595 (2015).

26. Watkins, E. B. \& Phillips, R. S. Inhibition of tyrosine phenol-lyase from Citrobacter freundii by 2-azatyrosine and 3-azatyrosine. Biochemistry 40 , 14862-14868 (2001)

27. Watkins, E. B. \& Phillips, R. S. Enzymatic synthesis of aza-l-tyrosines. Bioorg Med. Chem. Lett. 11, 2099-2100 (2001).

28. Bertin, C. et al. Grass roots chemistry: meta-tyrosine, an herbicidal nonprotein amino acid. Proc. Natl Acad. Sci. USA 104, 16964-16969 (2007).

29. Mishima, E. et al. Alteration of the intestinal environment by lubiprostone is associated with amelioration of adenine-induced CKD. J. Am. Soc. Nephrol. 26, 1787-1794 (2015).

30. Sato, E., et al. Impact of the oral adsorbent AST-120 on organ-specific accumulation of uremic toxins: LC-MS/MS and MS imaging techniques. Toxins 10, https://doi.org/10.3390/toxins10010019 (2017). 
31. Kumagai, H., Yamada, H., Matsui, H., Ohkishi, H. \& Ogata, K. Tyrosine phenol lyase. I. Purification, crystallization, and properties. J. Biol. Chem. 245 , 1767-1772 (1970).

32. Phillips, R. S., Demidkina, T. V. \& Faleev, N. G. The role of substrate strain in the mechanism of the carbon-carbon lyases. Bioorg. Chem. 57, 198-205 (2014).

33. Harris, A. P. \& Phillips, R. S. Benzimidazole analogs of (L)-tryptophan are substrates and inhibitors of tryptophan indole lyase from Escherichia coli. FEBS J. 280, 1807-1817 (2013).

34. Kikuchi, K. et al. Metabolomic analysis of uremic toxins by liquid chromatography/electrospray ionization-tandem mass spectrometry. J. Chromatogr. B Anal. Technol. Biomed. Life Sci. 878, 1662-1668 (2010).

35. Yamamoto, S. et al. Continuous reduction of protein-bound uraemic toxins with improved oxidative stress by using the oral charcoal adsorbent AST-120 in haemodialysis patients. Sci. Rep. 5, 14381 (2015).

36. Macisaac, R. J., Ekinci, E. I. \& Jerums, G. Markers of and risk factors for the development and progression of diabetic kidney disease. Am. J. Kidney Dis. 63, S39-S62 (2014).

37. Parving, H. H., Persson, F. \& Rossing, P. Microalbuminuria: a parameter that has changed diabetes care. Diabetes Res. Clin. Pract. 107, 1-8 (2015).

38. Lorenzo, V., Saracho, R., Zamora, J., Rufino, M. \& Torres, A. Similar renal decline in diabetic and non-diabetic patients with comparable levels of albuminuria. Nephrol. Dial. Transplant. 25, 835-841 (2010).

39. Reidy, K., Kang, H. M., Hostetter, T. \& Susztak, K. Molecular mechanisms of diabetic kidney disease. J. Clin. Invest. 124, 2333-2340 (2014).

40. MacIsaac, R. J., Ekinci, E. I. \& Jerums, G. Progressive diabetic nephropathy. How useful is microalbuminuria?: contra. Kidney Int. 86, 50-57 (2014).

41. Wang, Z. et al. Gut flora metabolism of phosphatidylcholine promotes cardiovascular disease. Nature 472, 57-63 (2011)

42. Kalantar-Zadeh, K. \& Fouque, D. Nutritional management of chronic kidney disease. N. Engl. J. Med. 377, 1765-1776 (2017).

43. Niwa, T., Ise, M. \& Miyazaki, T. Progression of glomerular sclerosis in experimental uremic rats by administration of indole, a precursor of indoxyl sulfate. Am. J. Nephrol. 14, 207-212 (1994).

44. Konishi, K. et al. AST-120 (Kremezin) initiated in early stage chronic kidney disease stunts the progression of renal dysfunction in type 2 diabetic subjects. Diabetes Res. Clin. Pract. 81, 310-315 (2008).

45. Ising, C. et al. Prohibitin-2 depletion unravels extra-mitochondrial functions at the kidney filtration barrier. Am. J. Pathol. 186, 1128-1139 (2016).

46. Suzuki, T. et al. Mitochonic acid 5 binds mitochondria and ameliorates renal tubular and cardiac myocyte damage. J. Am. Soc. Nephrol. 27, 1925-1932 (2016).

47. Zeier, M. \& Reiser, J. suPAR and chronic kidney disease-a podocyte story. Pflug. Arch. 469, 1017-1020 (2017).

48. Qin, J. et al. A metagenome-wide association study of gut microbiota in type 2 diabetes. Nature 490, 55-60 (2012).

49. Vaziri, N. D., Zhao, Y. Y. \& Pahl, M. V. Altered intestinal microbial flora and impaired epithelial barrier structure and function in CKD: the nature, mechanisms, consequences and potential treatment. Nephrol. Dial. Transplant. 31, 737-746 (2016).

50. Vaziri, N. D. et al. Disintegration of colonic epithelial tight junction in uremia: a likely cause of CKD-associated inflammation. Nephrol. Dial. Transpl. 27, 2686-2693 (2012).

51. Cani, P. D. et al. Changes in gut microbiota control metabolic endotoxemiainduced inflammation in high-fat diet-induced obesity and diabetes in mice. Diabetes 57, 1470-1481 (2008)

52. Mishima, E. et al. Canagliflozin reduces plasma uremic toxins and alters the intestinal microbiota composition in a chronic kidney disease mouse model. Am. J. Physiol. Renal Physiol. 315, F824-F833 (2018).

53. $\mathrm{Xu}, \mathrm{K}$. Y. et al. Impaired renal function and dysbiosis of gut microbiota contribute to increased trimethylamine- $\mathrm{N}$-oxide in chronic kidney disease patients. Sci. Rep. 7, 1445 (2017).

54. Kageyama, A. \& Benno, Y. Catenibacterium mitsuokai gen. nov., sp. nov., a gram-positive anaerobic bacterium isolated from human faeces. Int. J. Syst. Evol. Microbiol. 50(Pt 4), 1595-1599 (2000).

55. Gevers, D. et al. The treatment-naive microbiome in new-onset Crohn's disease. Cell Host Microbe 15, 382-392 (2014).

56. Bone, E., Tamm, A. \& Hill, M. The production of urinary phenols by gut bacteria and their possible role in the causation of large bowel cancer. Am. J. Clin. Nutr. 29, 1448-1454 (1976).

57. McDonald, T. A., Holland, N. T., Skibola, C., Duramad, P. \& Smith, M. T Hypothesis: phenol and hydroquinone derived mainly from diet and gastrointestinal flora activity are causal factors in leukemia. Leukemia 15, 10-20 (2001).
58. Hsiao, E. Y. et al. Microbiota modulate behavioral and physiological abnormalities associated with neurodevelopmental disorders. Cell $\mathbf{1 5 5}$, 1451-1463 (2013)

59. Saito, M. et al. N-hexacosanol ameliorates streptozotocin-induced diabetic rat nephropathy. Eur. J. Pharmacol. 544, 132-137 (2006).

60. Lopes, J. A. et al. Evaluation of pre-implantation kidney biopsies: comparison of Banff criteria to a morphometric approach. Kidney Int. 67, 1595-1600 (2005).

61. Shima, H. et al. A novel indole compound MA-35 attenuates renal fibrosis by inhibiting both TNF-alpha and TGF-betal pathways. Sci. Rep. 7, 1884 (2017).

62. Horio, M., Imai, E., Yasuda, Y., Watanabe, T. \& Matsuo, S. Modification of the CKD epidemiology collaboration (CKD-EPI) equation for Japanese: accuracy and use for population estimates. Am. J. Kidney Dis. 56, 32-38 (2010).

63. Kropelin, T. F. et al. Determining the optimal protocol for measuring an albuminuria class transition in clinical trials in diabetic kidney disease. J. Am. Soc. Nephrol. 27, 3405-3412 (2016).

64. Schmieder, R. E. et al. Changes in albuminuria predict mortality and morbidity in patients with vascular disease. J. Am. Soc. Nephrol. 22, 1353-1364 (2011).

65. Kim, S. W. et al. Robustness of gut microbiota of healthy adults in response to probiotic intervention revealed by high-throughput pyrosequencing. DNA Res. 20, 241-253 (2013).

66. Magoc, T. \& Salzberg, S. L. FLASH: fast length adjustment of short reads to improve genome assemblies. Bioinformatics 27, 2957-2963 (2011).

67. Caporaso, J. G. et al. QIIME allows analysis of high-throughput community sequencing data. Nat. Methods 7, 335-336 (2010).

68. Benjamini, Y. \& Hochberg, Y. Controlling the false discovery rate: a practical and powerful approach to multiple. Test. J. R. Stat. Soc. Ser. B 57, 289-300 (1995).

69. Ihaka, R. \& Gentleman, R. R. A language for data analysis and graphics. J. Comput. Graph. Stat. 5, 299-314 (1996).

70. Morino, Y. \& Snell, E. Tryptophanase (Escherichia coli B). Methods Enzymol. 17 (Part A), 439-446 (1970).

\section{Acknowledgements}

We thank the following people. Akinori Yuri and Tatsuki Tachikawa (Laboratory of Oncology, Pharmacy Practice and Sciences, Graduate School of Pharmaceutical Sciences) and Kiyoki Kitagawa and Tomoaki Funamoto (Department of Nephrology and Laboratory Medicine, Kanazawa University) for discussion. Masafumi Sugitani (Ono Pharmaceutical Co.) for technical support in the Tg rat experiments. Kazuhiro Tanabe (LSI Medience Co.) for the technical establishment of alternative measuring of PS and other uremic toxins. Fumiko Date and Miki Yoshizawa (Histological platform, Tohoku University School of Medicine) for histological assistance. Kazuyuki Hida (National Hospital Organization Okayama Medical Center, Okayama, Japan), Tatsuaki Nakato (Okayama Saiseikai General Hospital, Okayama, Japan), Takashi Matsuoka (Kurashiki Central Hospital), Ikki Shimizu (The Sakakibara Heart Institute of Okayama, Okayama Japan), Tomokazu Nunoue (Tsuyama Chuo Hospital, Okayama, Japan), Katsuhiro Miyashita (Japanese Red Cross Okayama Hospital, Okayama, Japan), and Shinichiro Ando (Okayama City General Medical Center, Okayama, Japan) for the U-CARE study. We also appreciate Dr. Tania Bezak at Trinity College Dublin for English editing. This work was supported in part by the Japan Society for the Promotion of Science KAKENHI Grant Numbers 18H02822 (T.A.), 23790168 (H.Y.), 15H00485 (T.S.), 16H04901 (S.F.) 17 H05654 (S.F.), and 18H04805 (S.F.), by the JST PRESTO JPMJPR1537 (S.F.) and from a grant of the Japan Kidney Foundation. This work was also supported in part by the Tohoku Medical Megabank Project through the Ministry of Education, Culture, Sports, Science and Technology, Japan; by the Reconstruction Agency, Japan; by the Japan Agency for Medical Research and Development (AMED; grant numbers JP18km0105001 and JP18km0105002) and also in part by the Intramural Research Program, NIDDK, NIH (J.B.K). T.S., F.N., and H.-J.H. were funded by a collaborative research grant of DSP.

\section{Author contributions}

K.K. and T.A. designed the experiment. D.S., Y.K., Y.M., and K.A. measured PS and uremic toxins. H.Y. and O.J. performed the uptake studies. K.K., S.N., H.-J.H., F.N., T.T., Y.O., C.C., Y.O., and S.S. performed the animal experiments. Y.M. synthesized the TPL inhibitors. K.K., M.B., and J.H. performed the human podocyte experiment. R.S. and D.S carried out the imaging MS and quantitative MS experiments. S.F., T.T., S.T., and N.-N.F performed the microbiome analysis. P.T. and N.S. performed untargeted LC/MS/MSbased metabolome analysis. Y.K., Y.A., and C.M. measured PS, IS, PCS, and TMAO by LC/MS/MS. K.M. and J.W. collected human samples (the U-CARE Study). T.I. and Y.O did Western blotting. Y.O. and N.T. completed the eNOS/Akita KO mice experiment. Y.O. and T.S. carried out histological analysis. T.S., E.M., H.S., Y.S., Y.A., T.M., Y.O., A.M., Y.O., M.I., and M.S. performed cell-based analysis and histological analysis. K.K. H.-J.H., K.T., and K.Y. measured TIL enzymatic activity. T.N. and A.H. did statistical analysis. J.B.K., S.K., S.O., N.M., T.W., S.I., T.Y., M.Y., and T.A. contributed to writing the discussion. 


\section{Additional information}

Supplementary Information accompanies this paper at https://doi.org/10.1038/s41467019-09735-4.

Competing interests: The authors declare no competing interests.

Reprints and permission information is available online at http://npg.nature.com/ reprintsandpermissions/

Journal peer review information: Nature Communications thanks the anonymous reviewers for their contributions to the peer review of this work.

Publisher's note: Springer Nature remains neutral with regard to jurisdictional claims in published maps and institutional affiliations. (c) (i) Open Access This article is licensed under a Creative Commons Attribution 4.0 International License, which permits use, sharing, adaptation, distribution and reproduction in any medium or format, as long as you give appropriate credit to the original author(s) and the source, provide a link to the Creative Commons license, and indicate if changes were made. The images or other third party material in this article are included in the article's Creative Commons license, unless indicated otherwise in a credit line to the material. If material is not included in the article's Creative Commons license and your intended use is not permitted by statutory regulation or exceeds the permitted use, you will need to obtain permission directly from the copyright holder. To view a copy of this license, visit http://creativecommons.org/ licenses/by/4.0/.

(c) The Author(s) 2019

Koichi Kikuchi1 ${ }^{1,221}$, Daisuke Saigusa ${ }^{3,21}$, Yoshitomi Kanemitsu ${ }^{4,21}$, Yotaro Matsumoto ${ }^{4,21}$, Paxton Thanai ${ }^{5}$, Naoto Suzuki ${ }^{4}$, Koki Mise ${ }^{6}{ }^{6}$, Hiroaki Yamaguchi ${ }^{7}$, Tomohiro Nakamura ${ }^{8}$, Kei Asaji ${ }^{4}$, Chikahisa Mukawa ${ }^{4}$, Hiroki Tsukamoto $\circledast^{4}$, Toshihiro Sato $\circledast^{7}$, Yoshitsugu Oikawa ${ }^{9}$, Tomoyuki Iwasaki ${ }^{1}$, Yuji Oe ${ }^{10}$, Tomoya Tsukimi ${ }^{11}$, Noriko N. Fukuda ${ }^{11}$, Hsin-Jung HO ${ }^{1,12}$, Fumika Nanto-Hara, ${ }^{12}$, Jiro Ogura', Ritsumi Saito ${ }^{3}$, Shizuko Nagao ${ }^{13}$, Yusuke Ohsaki', Satoshi Shimada (1) ', Takehiro Suzuki, 12, Takafumi Toyohara', Eikan Mishima', Hisato Shima (1) ${ }^{1}$, Yasutoshi Akiyama', Yukako Akiyama', Mariko Ichijo', Tetsuro Matsuhashi',12, Akihiro Matsuo', Yoshiaki Ogata², Ching-Chin Yang ${ }^{1,12}$, Chitose Suzuki', Matthew C. Breeggemann $\odot^{14}$, Jurgen Heymann ${ }^{14}$, Miho Shimizu ${ }^{15}$, Susumu Ogawa', Nobuyuki Takahashi ${ }^{10}$, Takashi Suzuki ${ }^{16}$, Yuji Owada $^{17}$, Shigeo Kure ${ }^{9}$, Nariyasu Mano ${ }^{7}$, Tomoyoshi Soga ${ }^{11}$, Takashi Wada ${ }^{15}$, Jeffrey B. Kopp ${ }^{14}$, Shinji Fukuda ${ }^{11,18,19,20}$, Atsushi Hozawa ${ }^{8}$, Masayuki Yamamoto ${ }^{3}$, Sadayoshi Ito', Jun Wada ${ }^{6}$, Yoshihisa Tomioka ${ }^{4} \&$ Takaaki Abe ${ }^{1}{ }^{1,2,12}$

1Division of Nephrology, Endocrinology and Vascular Medicine, Tohoku University Graduate School of Medicine, Sendai 980-8574, Japan.

${ }^{2}$ Department of Clinical Biology and Hormonal Regulation, Tohoku University Graduate School of Medicine, Sendai 980-8574, Japan. ${ }^{3}$ Department of Integrative Genomics, Tohoku Medical Megabank Organization, Tohoku University, Sendai 980-8573, Japan. ${ }^{4}$ Laboratory of Oncology, Pharmacy Practice and Sciences, Graduate School of Pharmaceutical Sciences, Sendai 980-8578, Japan. ${ }^{5}$ Waters Corporation, Tokyo 140-0001, Japan. ${ }^{6}$ Department of Nephrology, Rheumatology, Endocrinology and Metabolism, Okayama University Graduate School of Medicine, Dentistry and Pharmaceutical Sciences, Okayama 700-8558, Japan. ${ }^{7}$ Department of Pharmaceutical Sciences, Tohoku University Hospital, Sendai 980-8574, Japan. ${ }^{8}$ Department of Preventive Medicine and Epidemiology, Tohoku Medical Megabank Organization, Tohoku University, Sendai 980-8573, Japan. ${ }^{9}$ Department of Pediatrics, Tohoku University Graduate School of Medicine, Sendai 980-8574, Japan. ${ }^{10}$ Division of Clinical Pharmacology and Therapeutics, Tohoku University Graduate School of Pharmaceutical Sciences, Sendai 980-8578, Japan. ${ }^{11}$ Institute for Advanced Biosciences, Keio University, Tsuruoka 997-0052, Japan. ${ }^{12}$ Department of Medical Science, Tohoku University Graduate School of Biomedical Engineering, Sendai 980-8574, Japan. ${ }^{13}$ Education and Research Center of Animal Models for Human Diseases, Fujita Health University, Toyoake, Aichi 4701192, Japan. ${ }^{14}$ Kidney Diseases Branch, NIDDK, NIH, Bethesda, MD 20892-1268, USA. ${ }^{15}$ Department of Nephrology and Laboratory Medicine, Kanazawa University, Kanazawa 920-8641, Japan. ${ }^{16}$ Department of Pathology and Histotechnology, Tohoku University Graduate School of Medicine, Sendai 980-8574, Japan. ${ }^{17}$ Department of Organ Anatomy, Tohoku University Graduate School of Medicine, Sendai 980-8574, Japan. ${ }^{18}$ Intestinal Microbiota Project, Kanagawa Institute of Industrial Science and Technology, Kawasaki 210-0821, Japan. ${ }^{19}$ Transborder Medical Research Center, University of Tsukuba, Tsukuba 305-8577, Japan. ${ }^{20}$ PRESTO, Japan Science and Technology Agency, Kawaguchi 332-0012, Japan. ${ }^{21}$ These authors contributed equally: Koichi Kikuchi, Daisuke Saigusa, Yoshitomi Kanemitsu, Yotaro Matsumoto. 